K-Means clustering using JSD as a metric (File S9)

\title{
K-Means Silhouette
}

\begin{tabular}{|c|c|c|c|c|c|}
\hline $\mathrm{K}$ & $\begin{array}{l}\text { Max } \\
\text { silhouette }\end{array}$ & $\begin{array}{l}\text { Min } \\
\text { silhouette }\end{array}$ & $\begin{array}{l}\text { Avg } \\
\text { silhouette }\end{array}$ & $\begin{array}{l}\text { Median } \\
\text { silhouette }\end{array}$ & $\begin{array}{l}\text { SD } \\
\text { silhouette }\end{array}$ \\
\hline 3 & 0.141249 & -0.09691 & 0.01028 & -0.00062 & 0.057646 \\
\hline 4 & 0.103918 & -0.09033 & 0.00579 & 0.001644 & 0.033285 \\
\hline 5 & 0.08759 & -0.09336 & -0.0004 & -0.0001 & 0.033229 \\
\hline 6 & 0.082057 & -0.07874 & 0.000245 & 0.001184 & 0.033786 \\
\hline 7 & 0.120895 & -0.09818 & -0.00444 & -0.00609 & 0.045686 \\
\hline 8 & 0.120895 & -0.10985 & -0.00172 & -0.00426 & 0.044932 \\
\hline 9 & 0.13107 & -0.08058 & 0.0025 & -0.00195 & 0.042987 \\
\hline 10 & 0.13107 & -0.08058 & 0.003749 & 0.000333 & 0.042591 \\
\hline 11 & 0.13107 & -0.08138 & 0.005374 & 0.000895 & 0.037716 \\
\hline 12 & 0.13107 & -0.08138 & 0.005741 & 8.69E-05 & 0.038527 \\
\hline 13 & 0.13107 & -0.07063 & 0.005918 & 0 & 0.037756 \\
\hline 14 & 0.143011 & -0.09187 & 0.007357 & 0.003579 & 0.043728 \\
\hline 15 & 0.143011 & -0.09187 & 0.007382 & 0.00316 & 0.043621 \\
\hline 16 & 0.143011 & -0.09187 & 0.007756 & 0.00316 & 0.0431 \\
\hline 17 & 0.143965 & -0.07546 & 0.010633 & 0.003947 & 0.041135 \\
\hline 18 & 0.143965 & -0.07546 & 0.011154 & 0.004963 & 0.040367 \\
\hline 19 & 0.143965 & -0.07546 & 0.012238 & 0.005434 & 0.042012 \\
\hline 20 & 0.143965 & -0.07546 & 0.012508 & 0.006872 & 0.041685 \\
\hline 21 & 0.143965 & -0. & 0.012 & 0.004 & 0.043734 \\
\hline 22 & 0.143965 & -0.07546 & 0.01272 & 0.004944 & 0.043477 \\
\hline 23 & 0.143965 & -0.07546 & 0.01255 & 0.004899 & 0.043491 \\
\hline 24 & 0.143965 & -0.07546 & 0.012667 & 0.005071 & 0.043373 \\
\hline 25 & 0.143965 & -0.07546 & 0.013634 & 0.008142 & 0.041571 \\
\hline 26 & 0.143965 & -0.07546 & 0.01371 & 0.008026 & 0.041569 \\
\hline 27 & 0.143965 & -0.07546 & 0.01442 & 0.008347 & 0.041477 \\
\hline 28 & 0.143965 & -0.07546 & 0.014826 & 0.008994 & 0.041209 \\
\hline 29 & 0.143965 & -0.07546 & 0.014572 & 0.008345 & 0.041055 \\
\hline 30 & 0.143965 & -0.07546 & 0.014989 & 0.008528 & 0.040773 \\
\hline 31 & 0.143965 & -0.07546 & 0.015305 & 0.008675 & 0.040629 \\
\hline 32 & 0.143965 & -0.07546 & 0.015706 & 0.009441 & 0.040305 \\
\hline 33 & 0.143965 & -0.07546 & 0.015656 & 0.008994 & 0.040566 \\
\hline 34 & 0.143965 & -0.07546 & 0.015998 & 0.008675 & 0.040626 \\
\hline 35 & 0.143965 & -0.07546 & 0.01587 & 0.008528 & 0.040869 \\
\hline 36 & 0.143965 & -0.07546 & 0.016301 & 0.009686 & 0.040602 \\
\hline 37 & 0.143965 & -0.06892 & 0.017077 & 0.009686 & 0.039608 \\
\hline 38 & 0.143965 & -0.06892 & 0.01703 & 0.008994 & 0.039718 \\
\hline
\end{tabular}




\begin{tabular}{r|rrrrr}
$\mathbf{3 9}$ & 0.143965 & -0.06892 & 0.017308 & 0.009686 & 0.039601 \\
$\mathbf{4 0}$ & 0.141686 & -0.06702 & 0.017391 & 0.0093 & 0.039119 \\
$\mathbf{4 1}$ & 0.141686 & -0.06702 & 0.017266 & 0.009075 & 0.0389 \\
$\mathbf{4 2}$ & 0.139114 & -0.08095 & 0.018012 & 0.01336 & 0.040741 \\
$\mathbf{4 3}$ & 0.136873 & -0.07986 & 0.018225 & 0.012173 & 0.040404 \\
$\mathbf{4 4}$ & 0.136873 & -0.07491 & 0.018762 & 0.012173 & 0.039666 \\
$\mathbf{4 5}$ & 0.136873 & -0.07491 & 0.019863 & 0.012976 & 0.040143 \\
$\mathbf{4 6}$ & 0.136873 & -0.07491 & 0.019847 & 0.012976 & 0.040075 \\
$\mathbf{4 7}$ & 0.136873 & -0.07491 & 0.019998 & 0.013673 & 0.040025 \\
$\mathbf{4 8}$ & 0.136873 & -0.07491 & 0.020012 & 0.013157 & 0.04 \\
$\mathbf{4 9}$ & 0.136873 & -0.07491 & 0.020098 & 0.013157 & 0.040028 \\
$\mathbf{5 0}$ & 0.136873 & -0.07491 & 0.01973 & 0.012602 & 0.039997 \\
$\mathbf{5 1}$ & 0.136873 & -0.07491 & 0.019708 & 0.012173 & 0.040029 \\
$\mathbf{5 2}$ & 0.136873 & -0.07094 & 0.02078 & 0.013708 & 0.039687 \\
$\mathbf{5 3}$ & 0.136873 & -0.07094 & 0.020476 & 0.01444 & 0.03925 \\
$\mathbf{5 4}$ & 0.136873 & -0.07094 & 0.020583 & 0.013708 & 0.03948 \\
$\mathbf{5 5}$ & 0.136873 & -0.07094 & 0.020765 & 0.012717 & 0.039905 \\
$\mathbf{5 6}$ & 0.136873 & -0.06645 & 0.021201 & 0.012717 & 0.039326 \\
$\mathbf{5 7}$ & 0.136873 & -0.06645 & 0.020348 & 0.012173 & 0.038691 \\
$\mathbf{5 8}$ & 0.136873 & -0.06645 & 0.020443 & 0.011601 & 0.039075 \\
$\mathbf{5 9}$ & 0.136873 & -0.06645 & 0.020182 & 0.011427 & 0.039085 \\
$\mathbf{6 0}$ & 0.136873 & -0.06105 & 0.020761 & 0.011427 & 0.03837
\end{tabular}

Silhouette values have been computed using silhouette() function in $\mathrm{R}$, according to $\mathrm{K}$ clusters.

(For each observation $\mathrm{i}$, the silhouette width $\mathrm{s}(\mathrm{i})$ is defined as follows: Put $\mathrm{a}(\mathrm{i})=$ average dissimilarity between $\mathrm{i}$ and all other points of the cluster to which $i$ belongs (if $i$ is the only observation in its cluster, $s(i):=0$ without further calculations). For all other clusters $\mathrm{C}$, put $\mathrm{d}(\mathrm{i}, \mathrm{C})=$ average dissimilarity of $i$ to all observations of $\mathrm{C}$. The smallest of these $d(i, C)$ is $b(i):=\mid \min \_C d(i, C)$, and can be seen as the dissimilarity between $i$ and its "neighbor" cluster, i.e., the nearest one to which it does not belong. Finally, $s(i):=(b(i)-a(i)) / \max (a(i), b(i)))$. 


\section{Color legends}

\begin{tabular}{|c|c|c|c|}
\hline Author & Color & Author & Color \\
\hline Beaumont & \#FFFFFF & Marlowe & \#FFFFFF \\
\hline Beaumont_and_Fletcher & \#99CC00 & Marlowe_and_Nashe & \#FFFFFF \\
\hline Brandon_Samuel & \#FFFFFF & Marmion & \#FFFFFF \\
\hline Brome & \#FFFFFF & Marston & \#FFFFFF \\
\hline Campion & \#FFFFFF & Massinger & \#FFFFFF \\
\hline Carew & \#FFFFFF & Middleton & \#99CCFF \\
\hline Carey & \#FFFFFF & Middleton_and_Rowley & \#99CCFF \\
\hline Chapman & \#FF6600 & Munday & \#FFFFFF \\
\hline Chettle & \#FFFFFF & Nashe & \#FFFFFF \\
\hline Daniel & \#FFFFFF & Oxford & \#FFFFFF \\
\hline Davenant & \#FFFFFF & Peele & \#FFFFFF \\
\hline Davies_John & \#FFFFFF & Porter_Henry & \#FFFFFF \\
\hline Day & \#FFFFFF & Raleigh & \#FFFFFF \\
\hline Dekker & \#FFFFFF & Rowley & \#FFFFFF \\
\hline Donne & \#FFFFFF & Shakespeare & \#CC99FF \\
\hline Drayton & \#FFFFFF & Shakespeare_and_Fletcher & \#CC99FF \\
\hline Fletcher & \#99CC00 & Shakespeare_and_Middleton & \#CC99FF \\
\hline Fletcher_and_Massinger & \#99CC00 & Shakespeare_and_Wilkins & \#CC99FF \\
\hline Ford & \#FFFFFF & Shakespeare_and_others & \#CC99FF \\
\hline Goffe & \#FFFFFF & Shirley & \#FFFFFF \\
\hline Greene & \#FFFFFF & Sidney & \#FFFFFF \\
\hline Greville_Fulke & \#FFFFFF & Sidney_Mary & \#FFFFFF \\
\hline Haughton & \#FFFFFF & Southwell & \#FFFFFF \\
\hline Herbert_George & \#FFFFFF & Spenser & \#FFFFFF \\
\hline Herbert_Mary & \#FFFFFF & Suckling & \#FFFFFF \\
\hline Herrick & \#FFFFFF & Tourneur & \#FFFFFF \\
\hline Heywood & \#FFFFFF & Uncertain & \#FF0000 \\
\hline Jonson & \#008000 & Unknown & \#FF0000 \\
\hline Kyd & \#FFFFFF & Webster & \#FFFFFF \\
\hline Lanyer & \#FFFFFF & Wilmot & \#FFFFFF \\
\hline Lodge & \#FFFFFF & Wilson & \#FFFFFF \\
\hline Lyly & \#FFFFFF & Wroth_Lady Mary & \#FFFFFF \\
\hline Markham_and_Sampson & \#FFFFFF & & \\
\hline
\end{tabular}


Graphical representations of K-Means clustering (for K = 3 to 60)
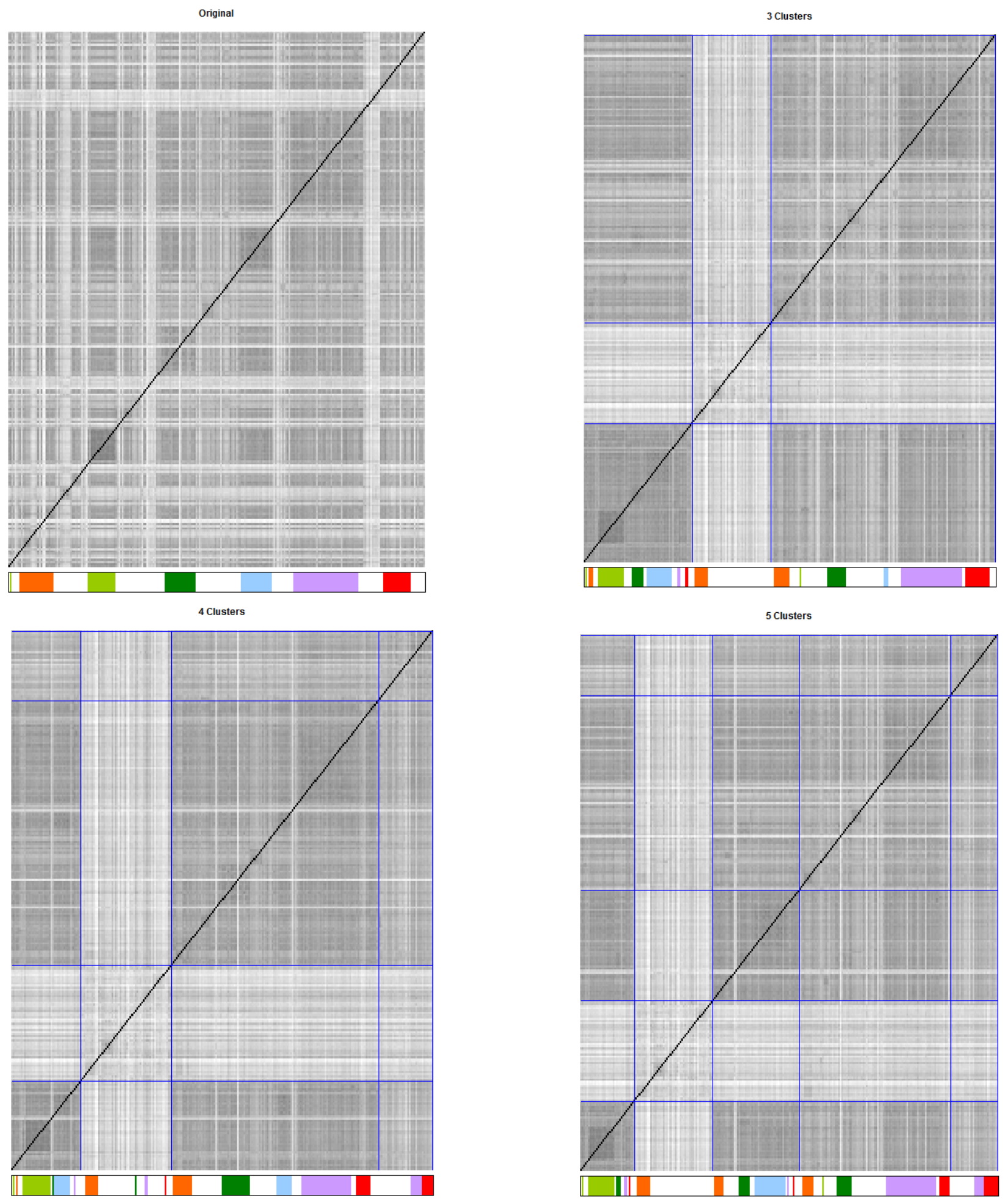

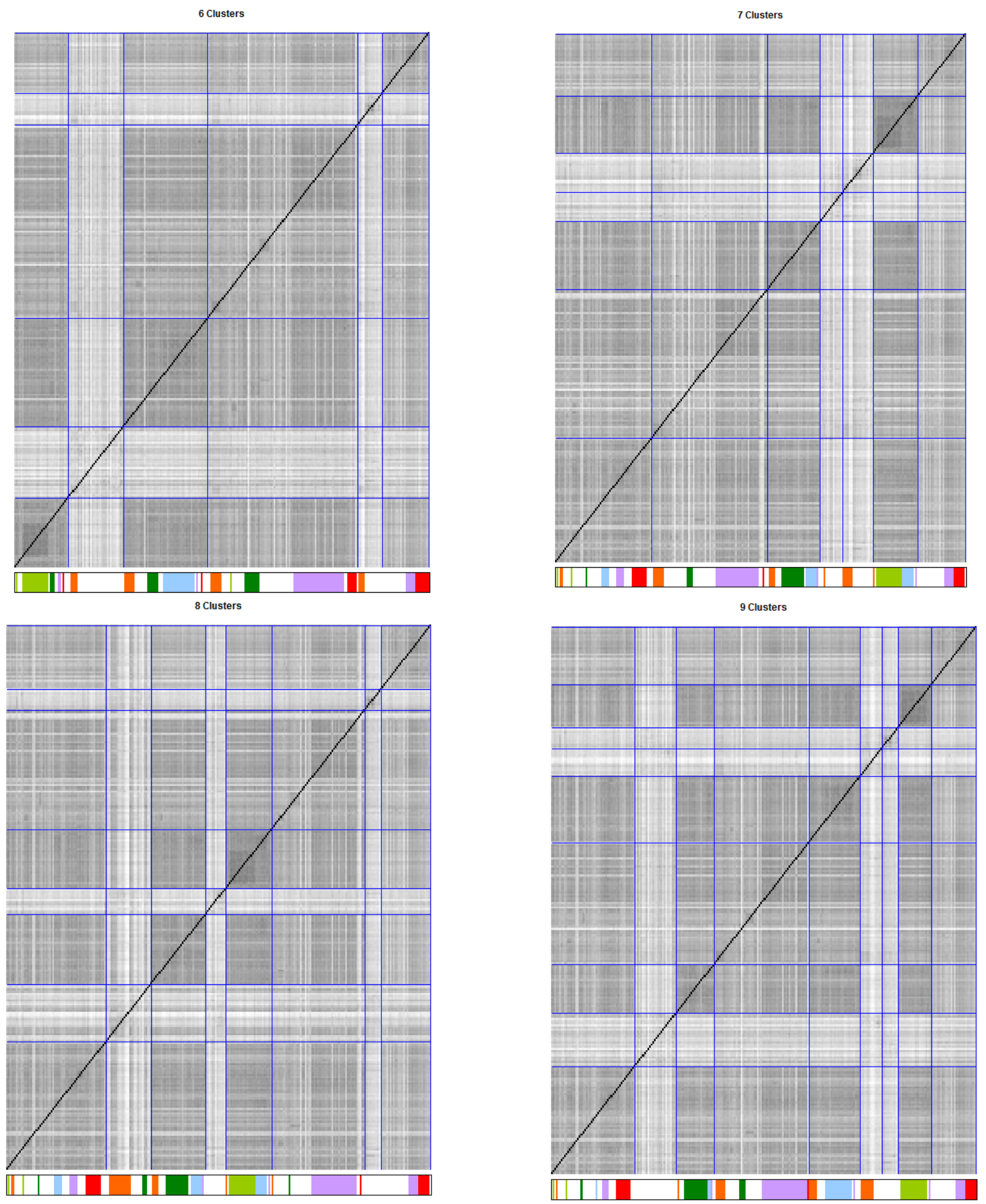


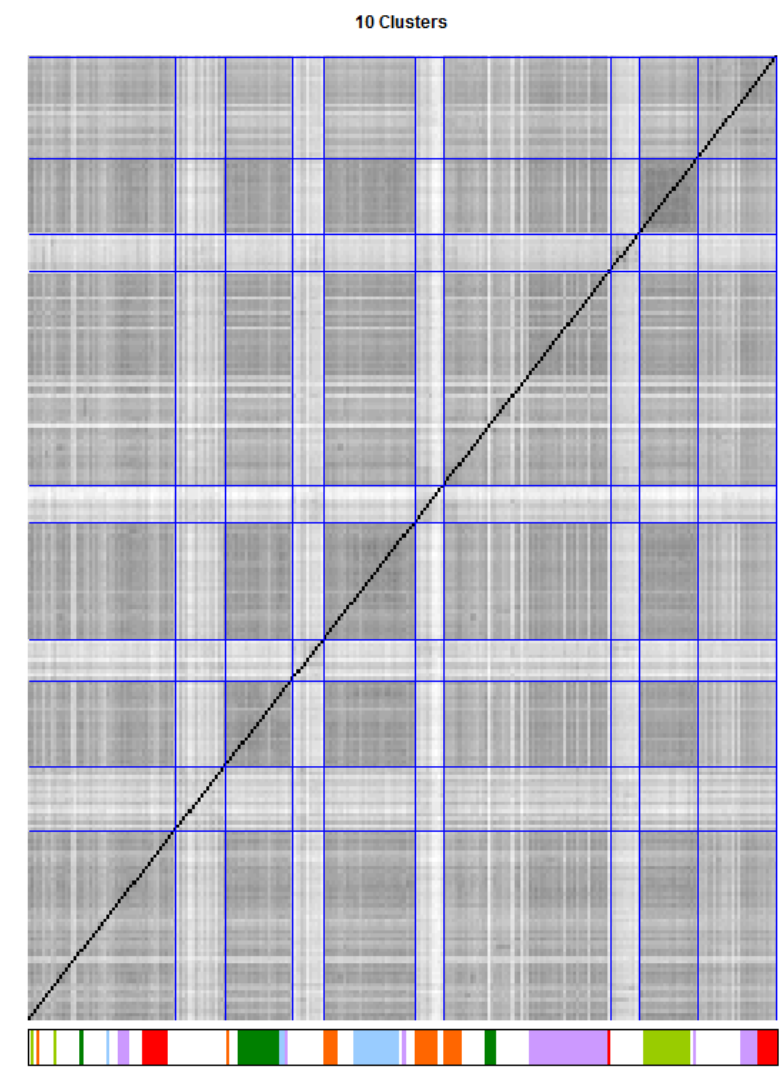

12 Clusters

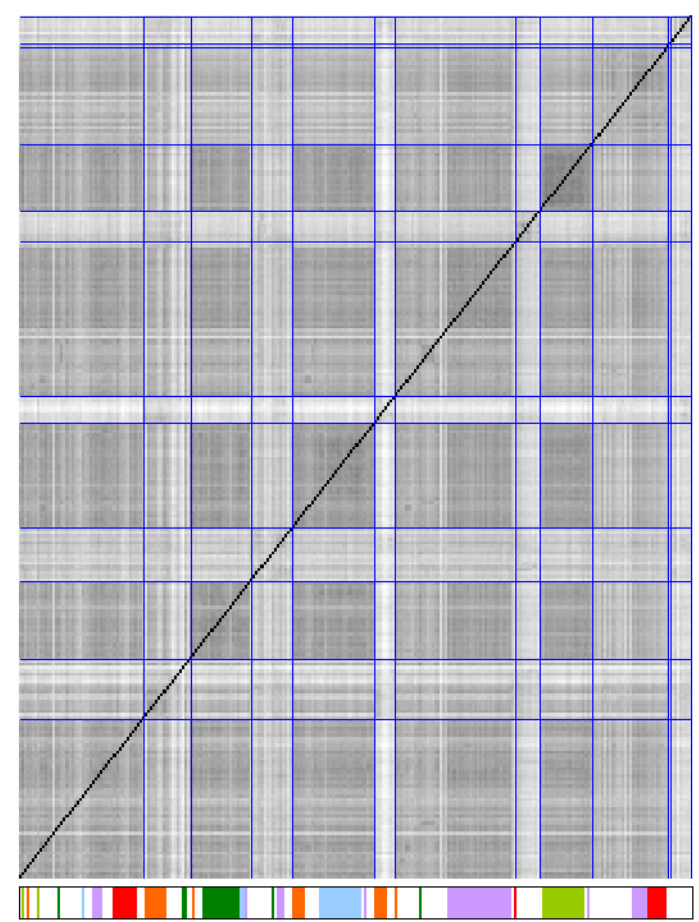

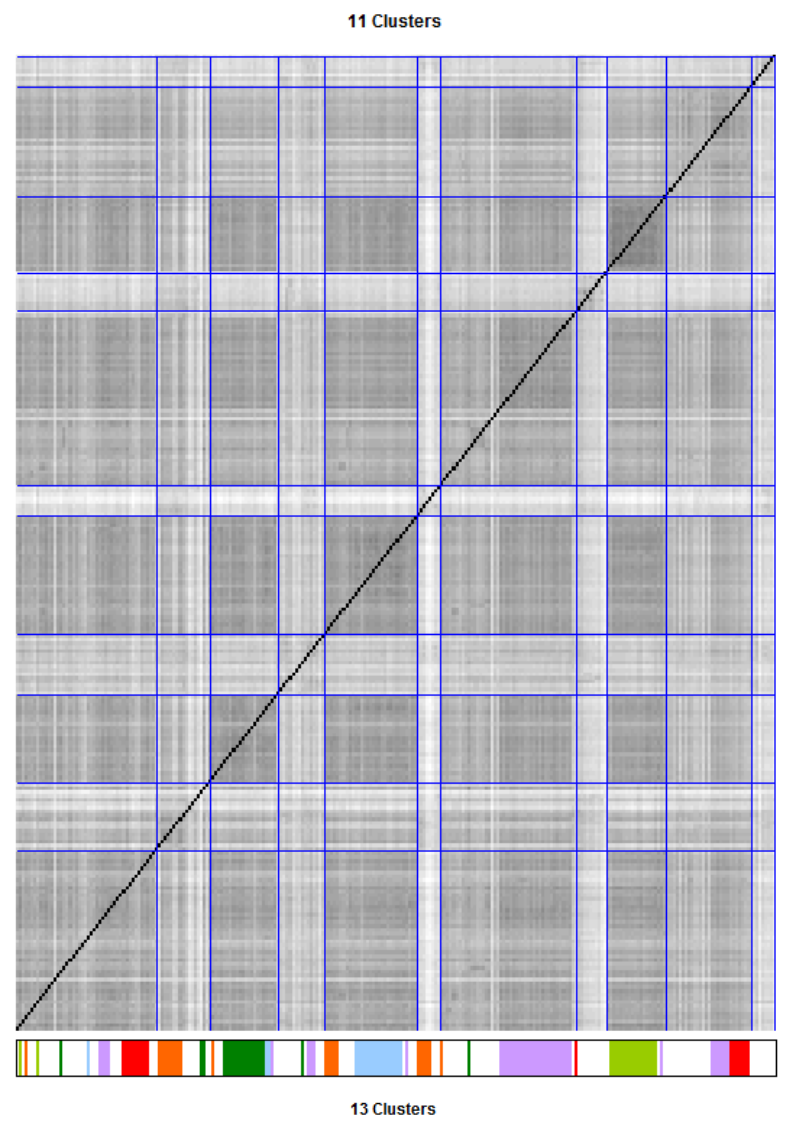

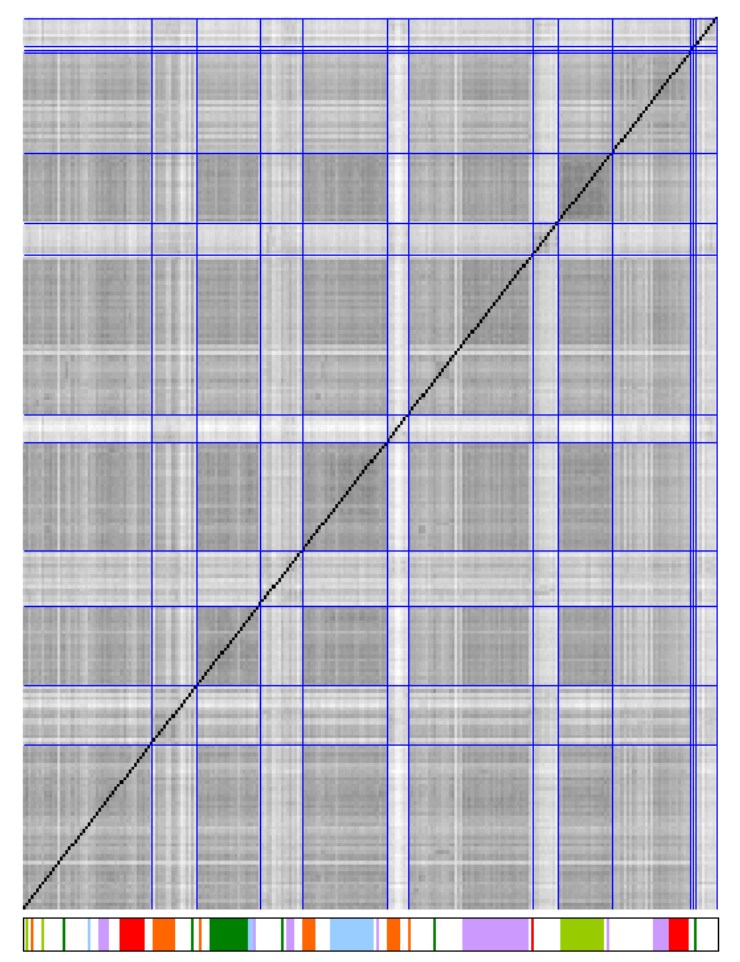


14 Clusters
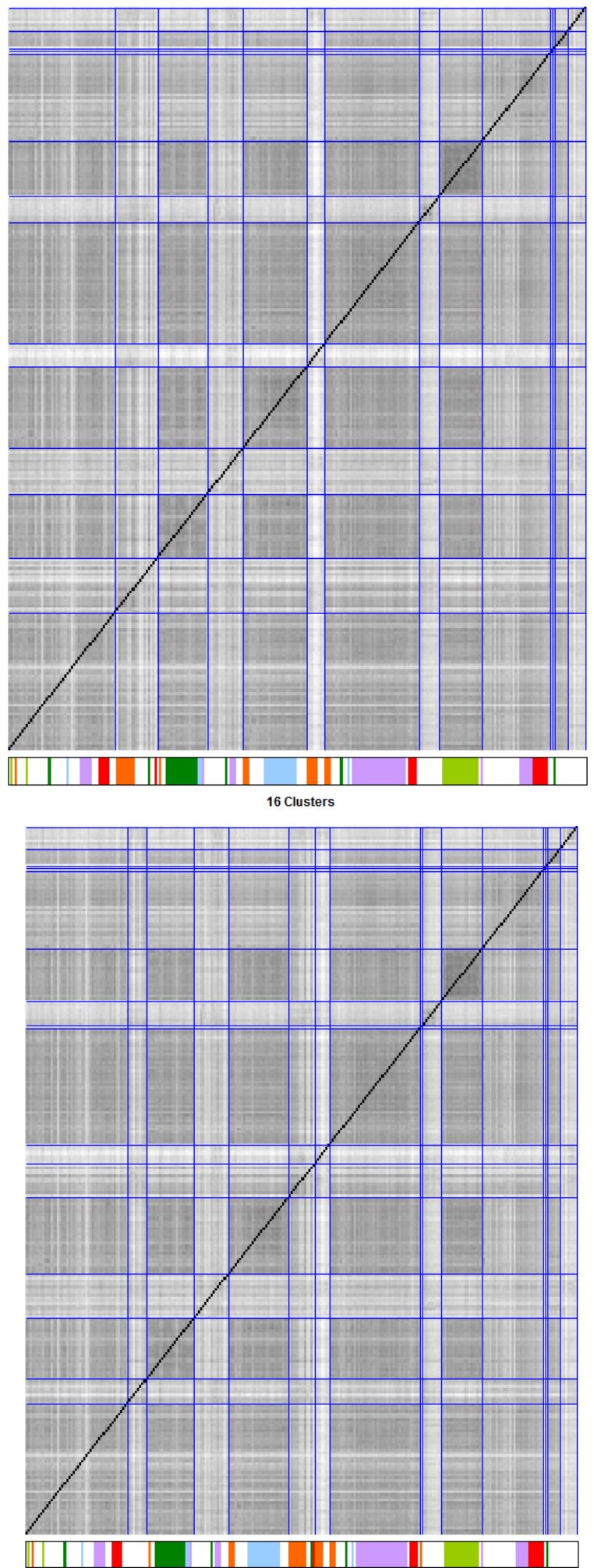

15 Clusters

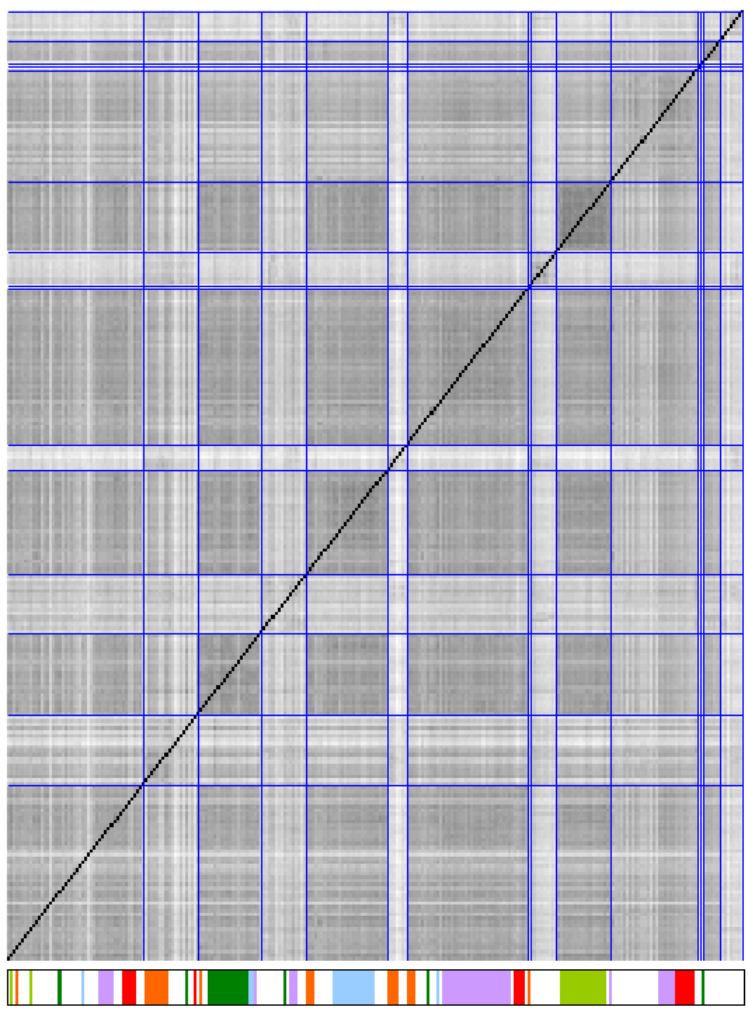

17 Clusters

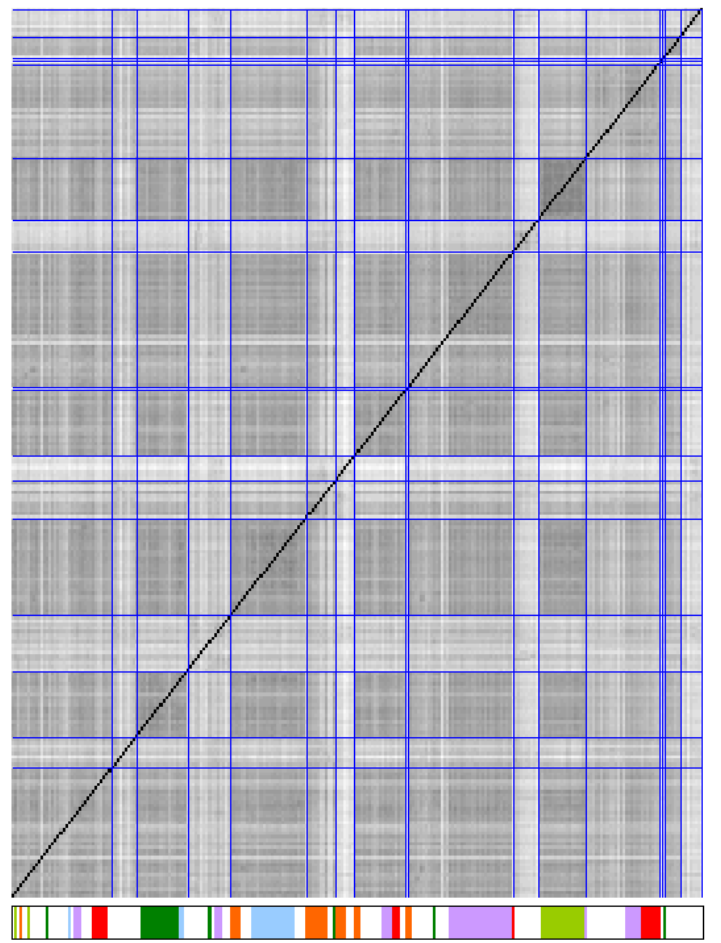


18 Clusters

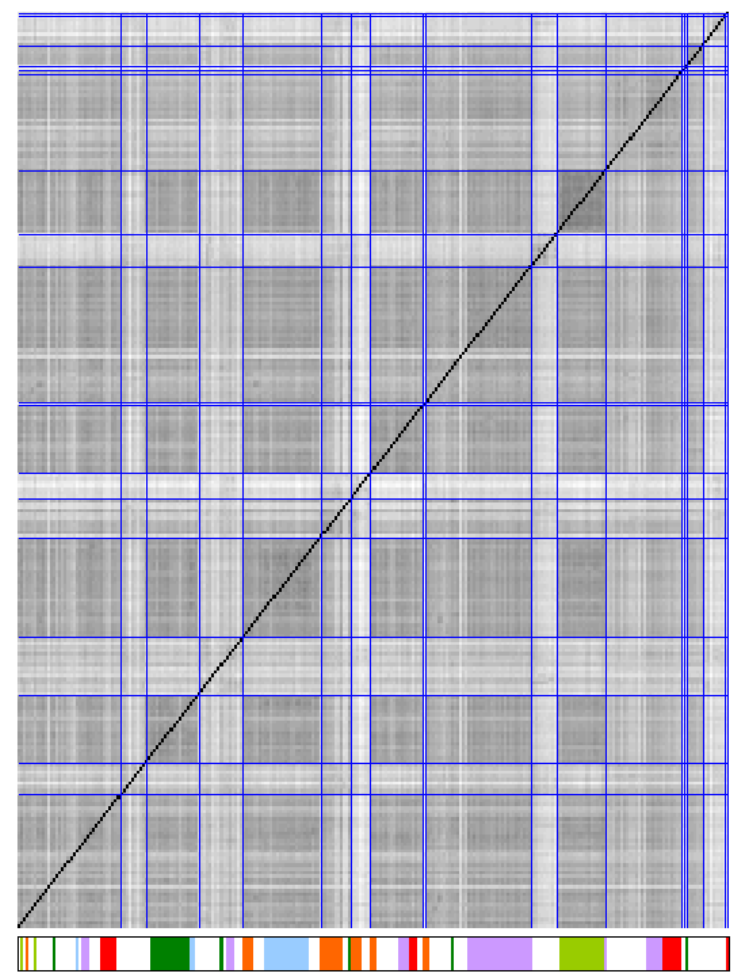

20 Clusters

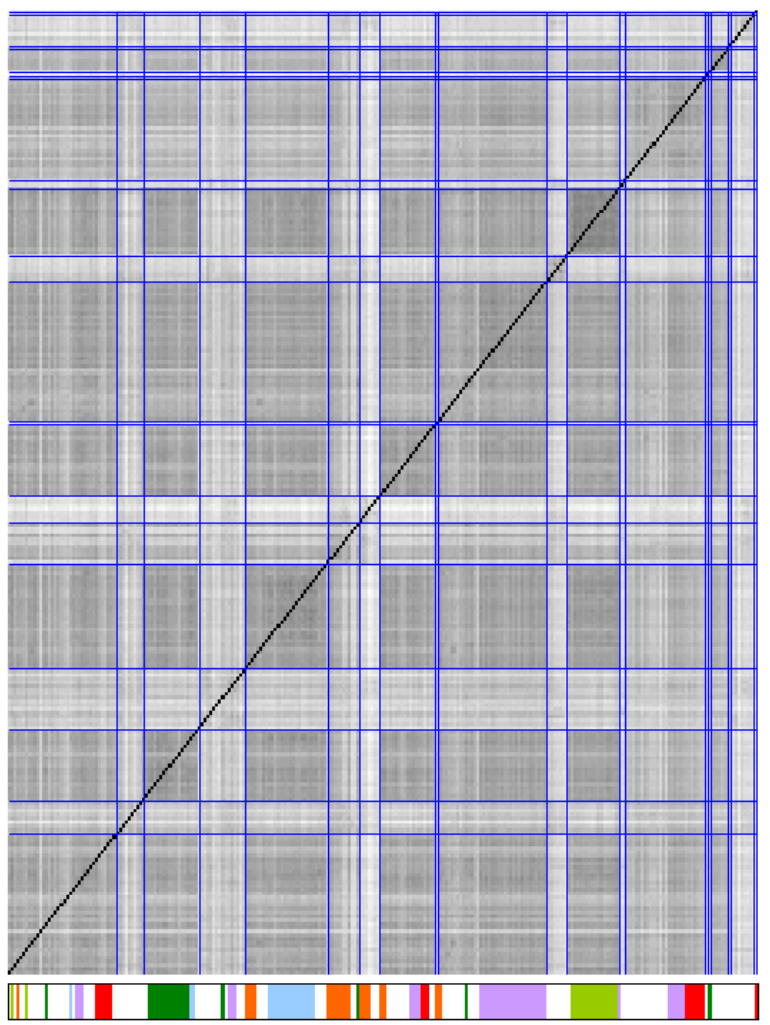

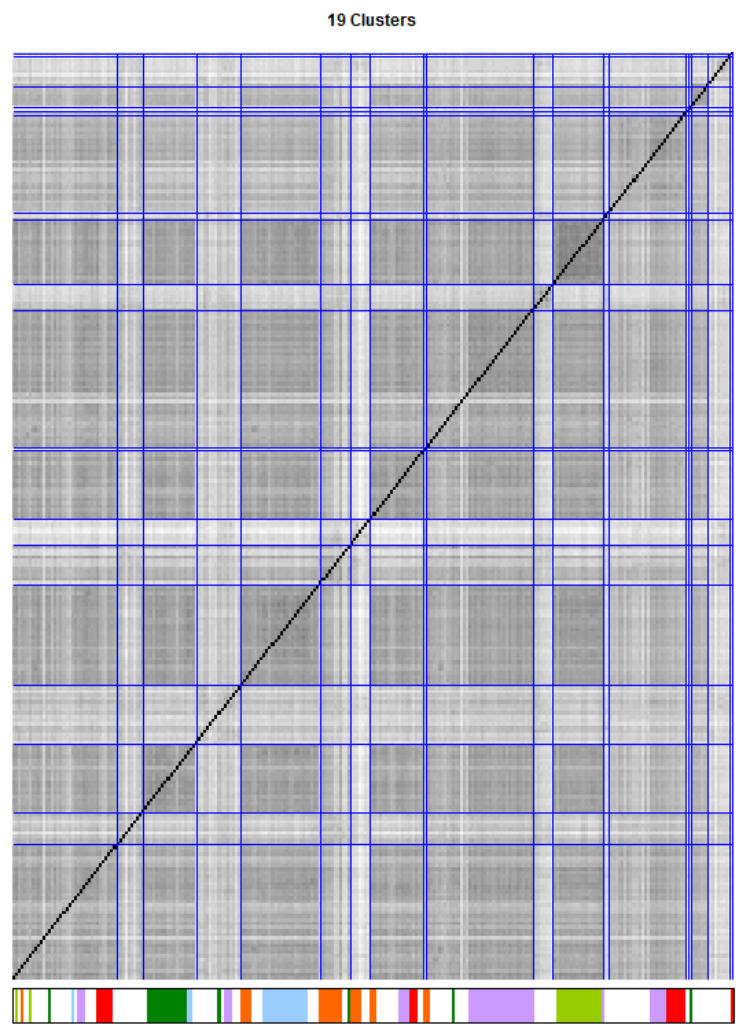

21 Clusters

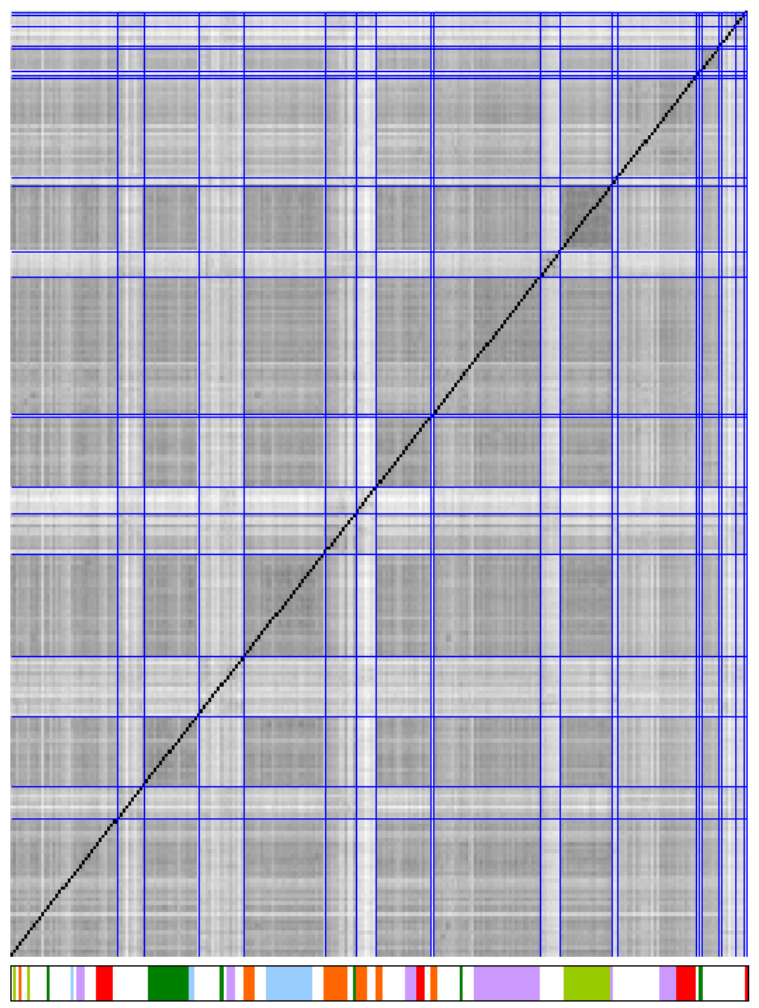


22 Clusters
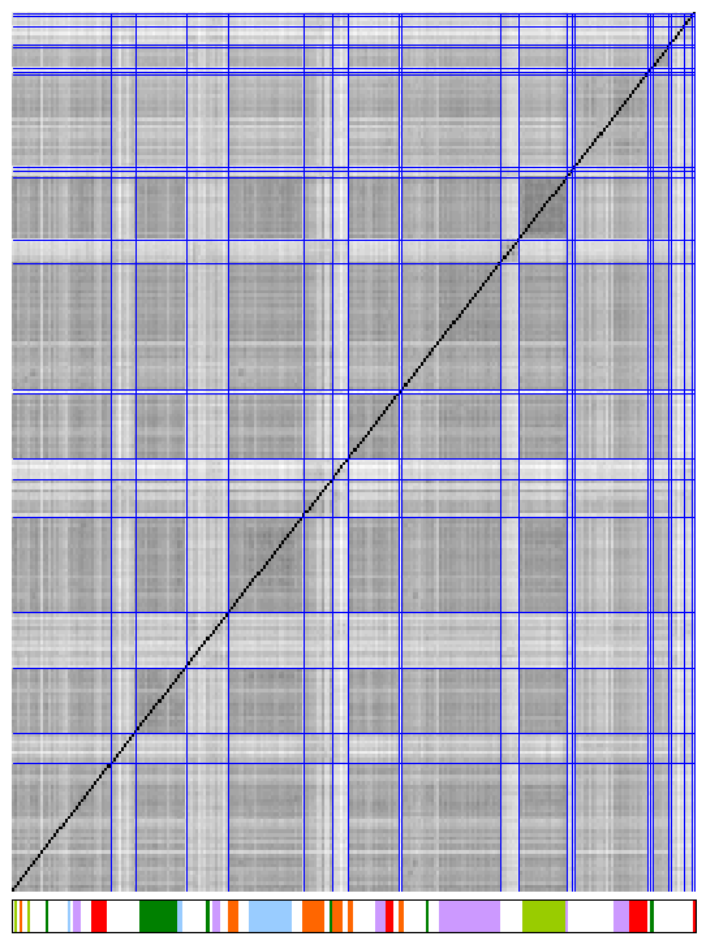

24 Clusters

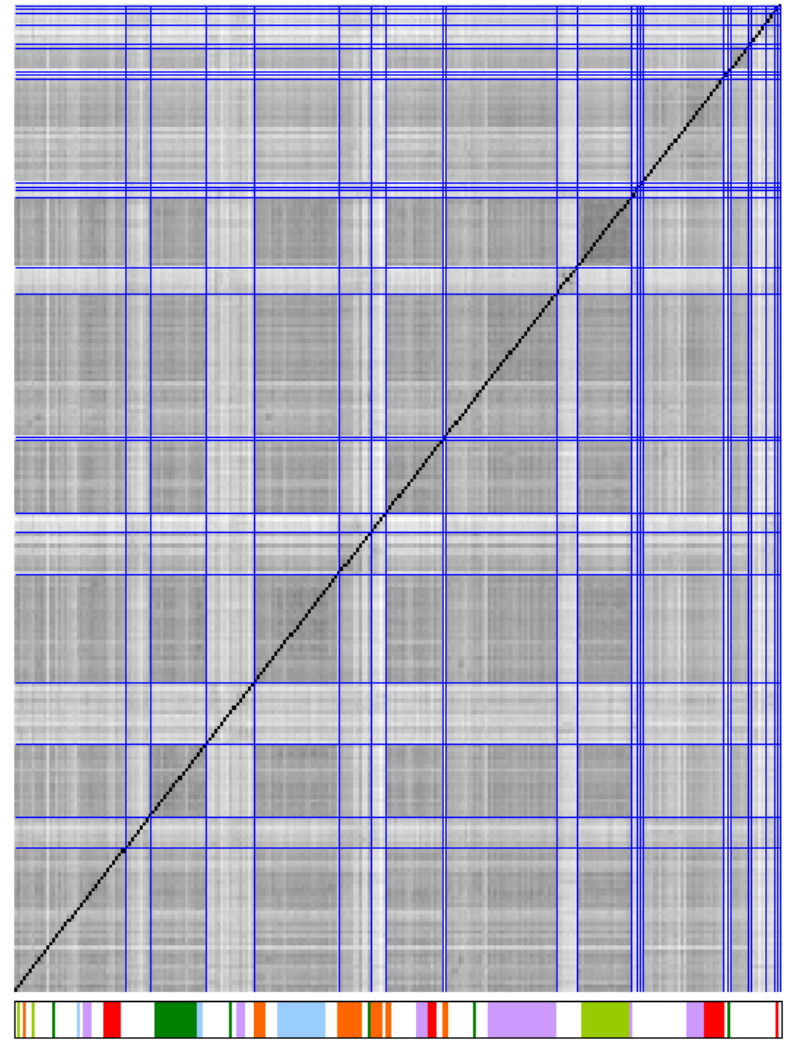

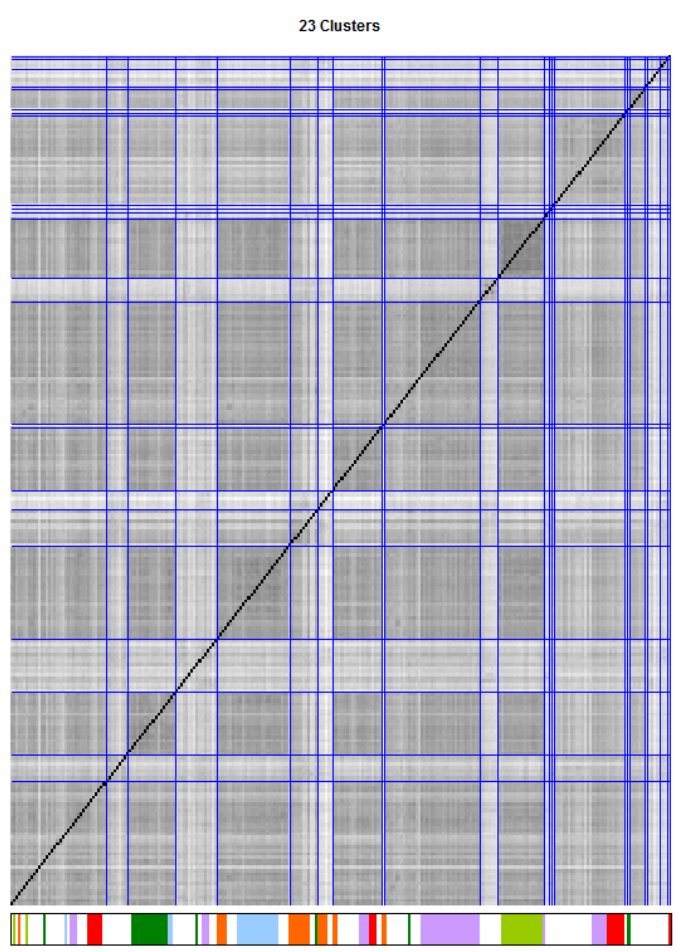

25 Clusters

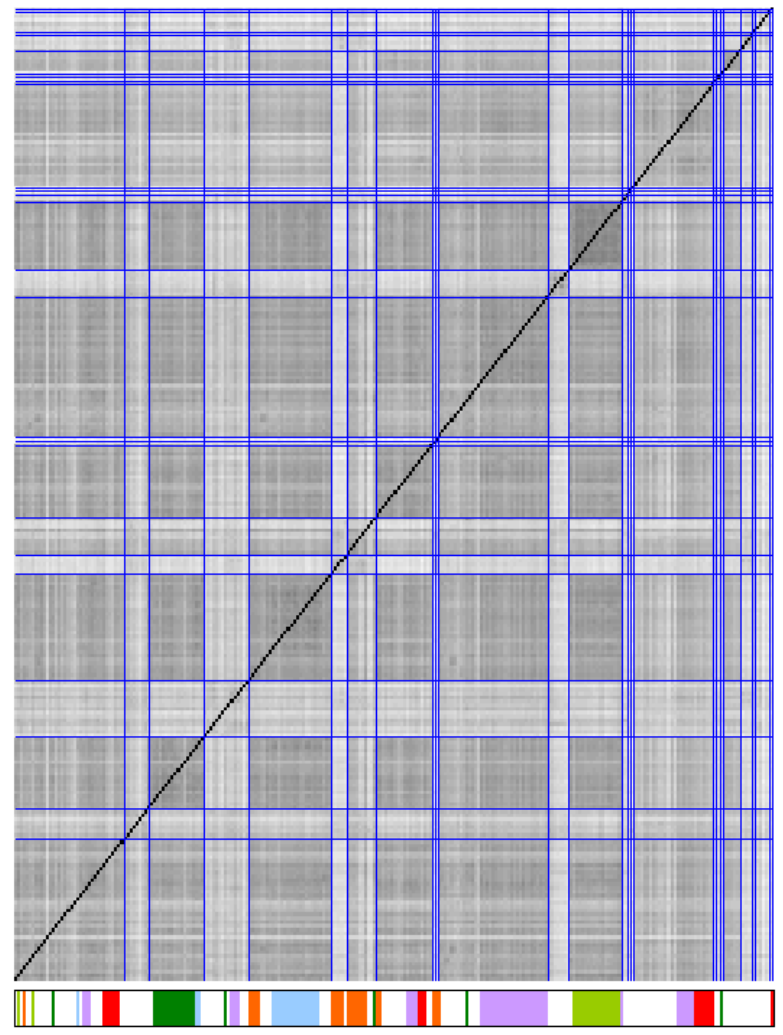


26 Clusters

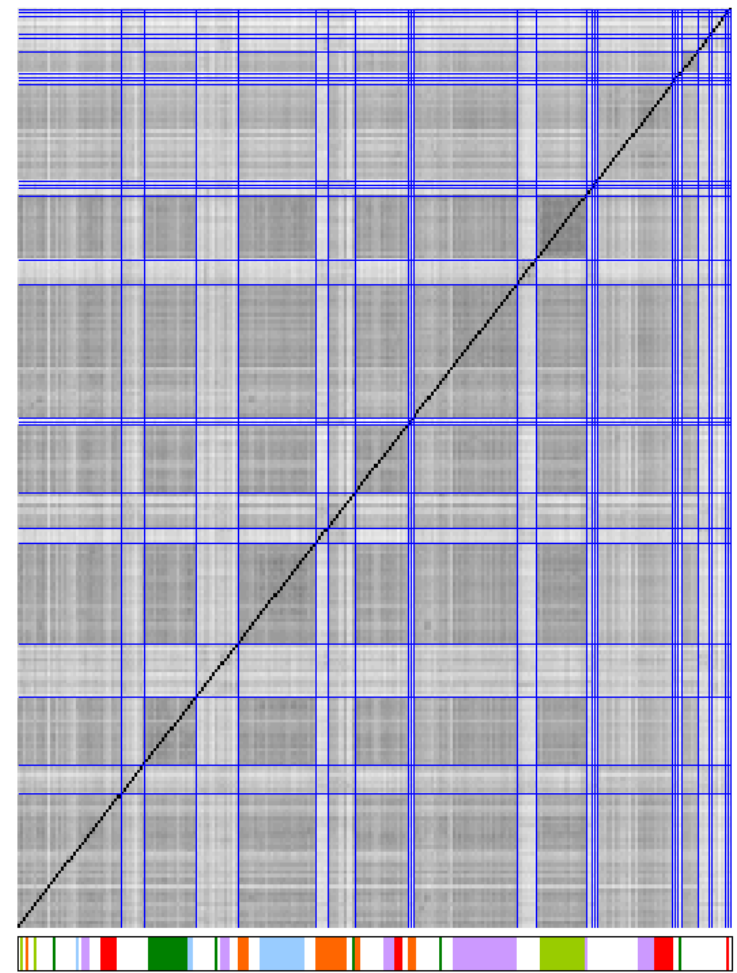

28 Clusters

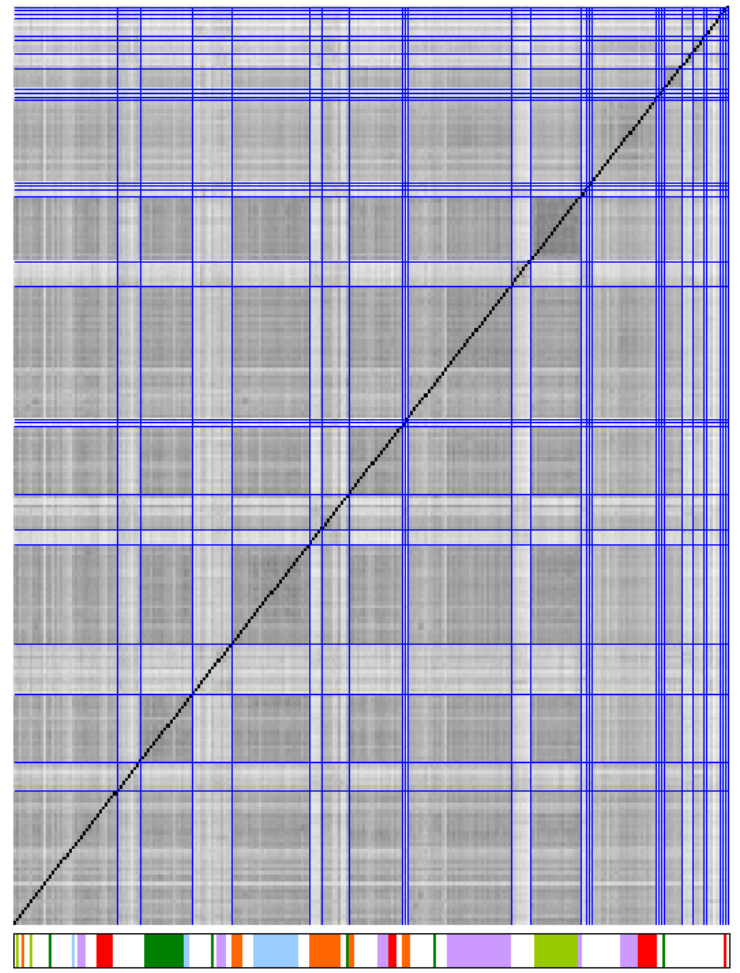

27 Clusters

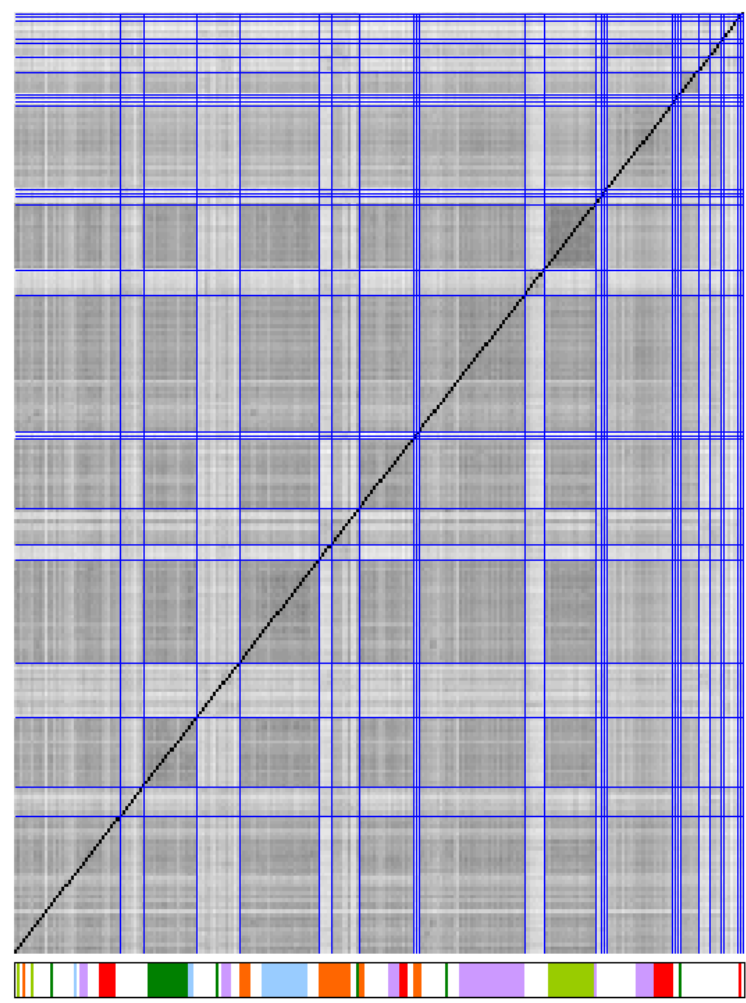

29 Clusters

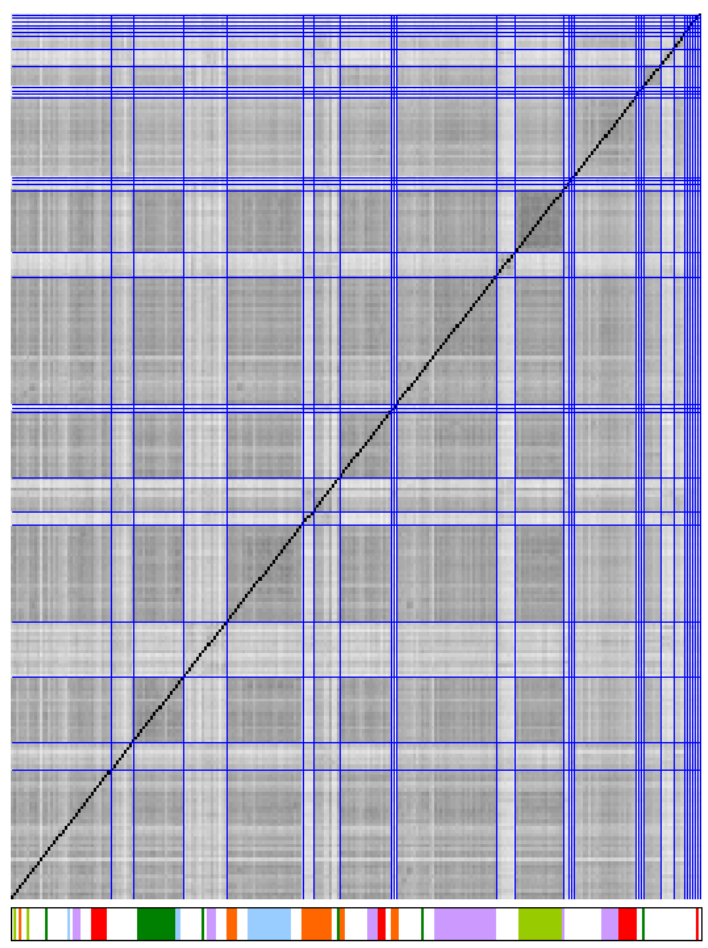


30 Clusters

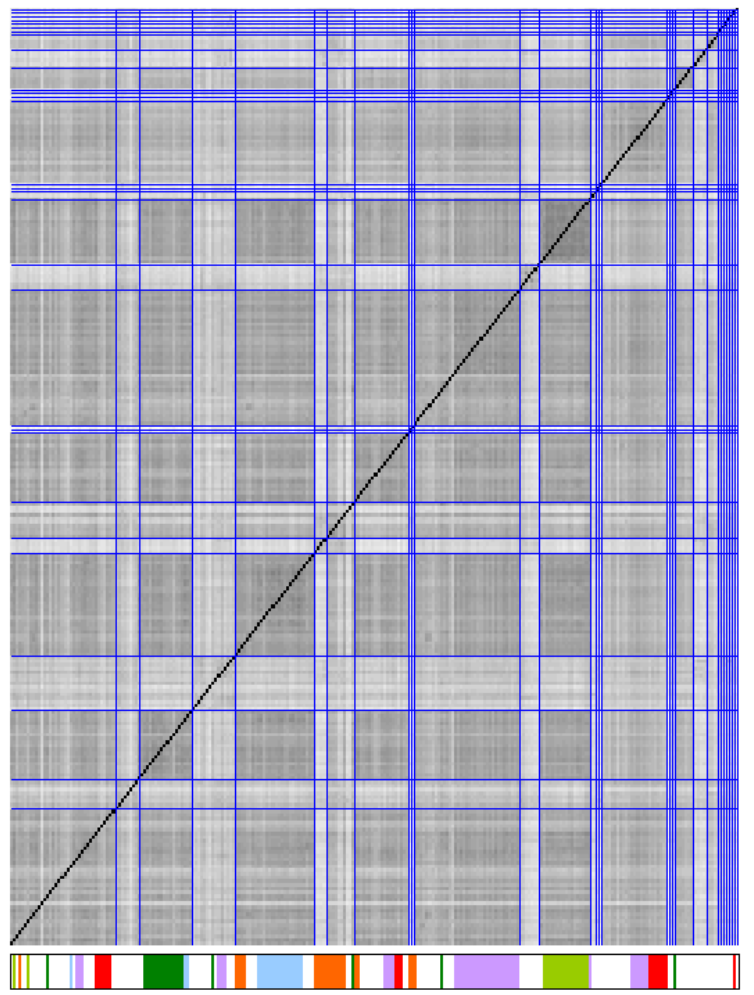

32 Clusters

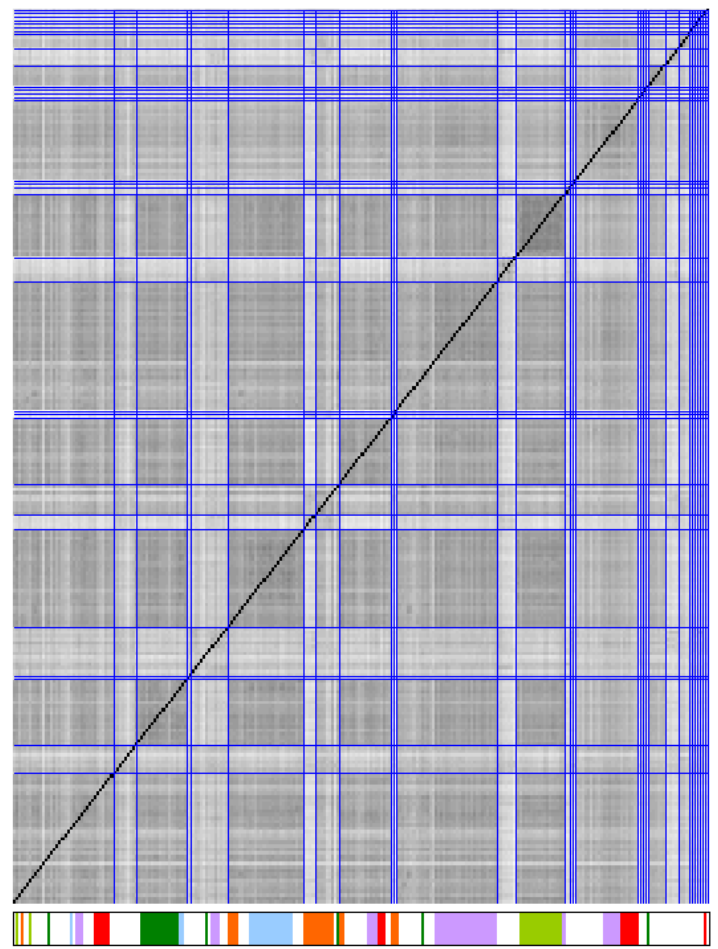

31 Clusters

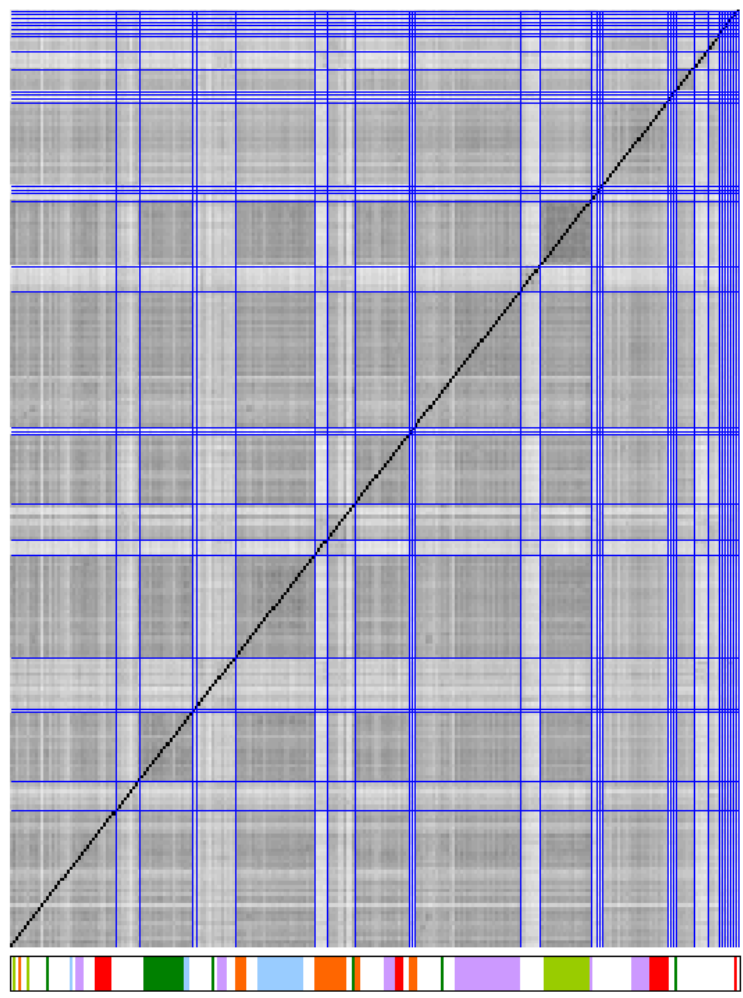

33 Clusters

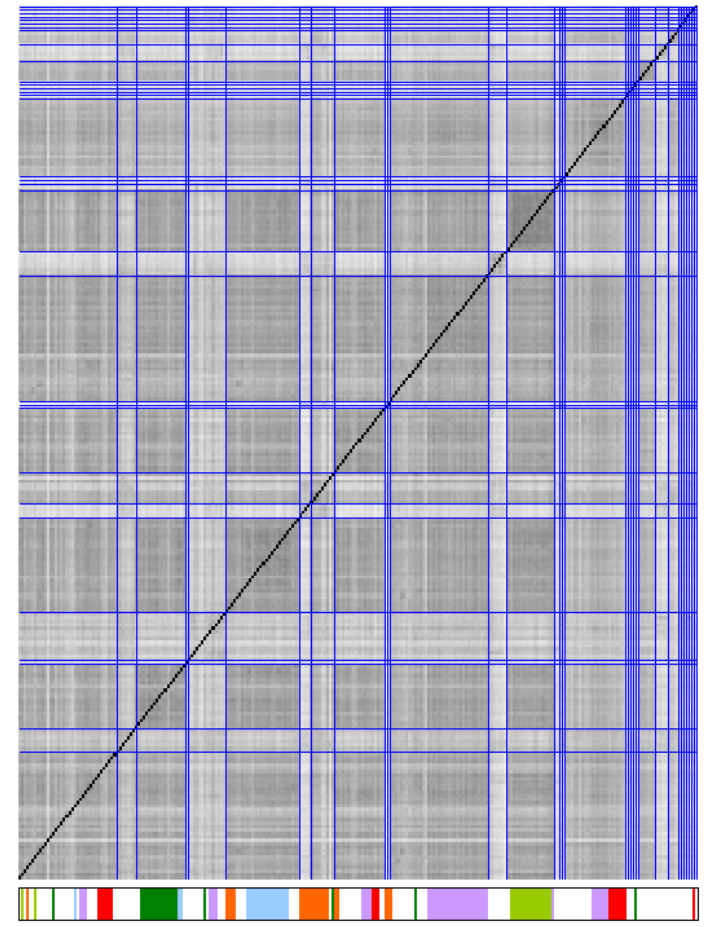


34 Clusters
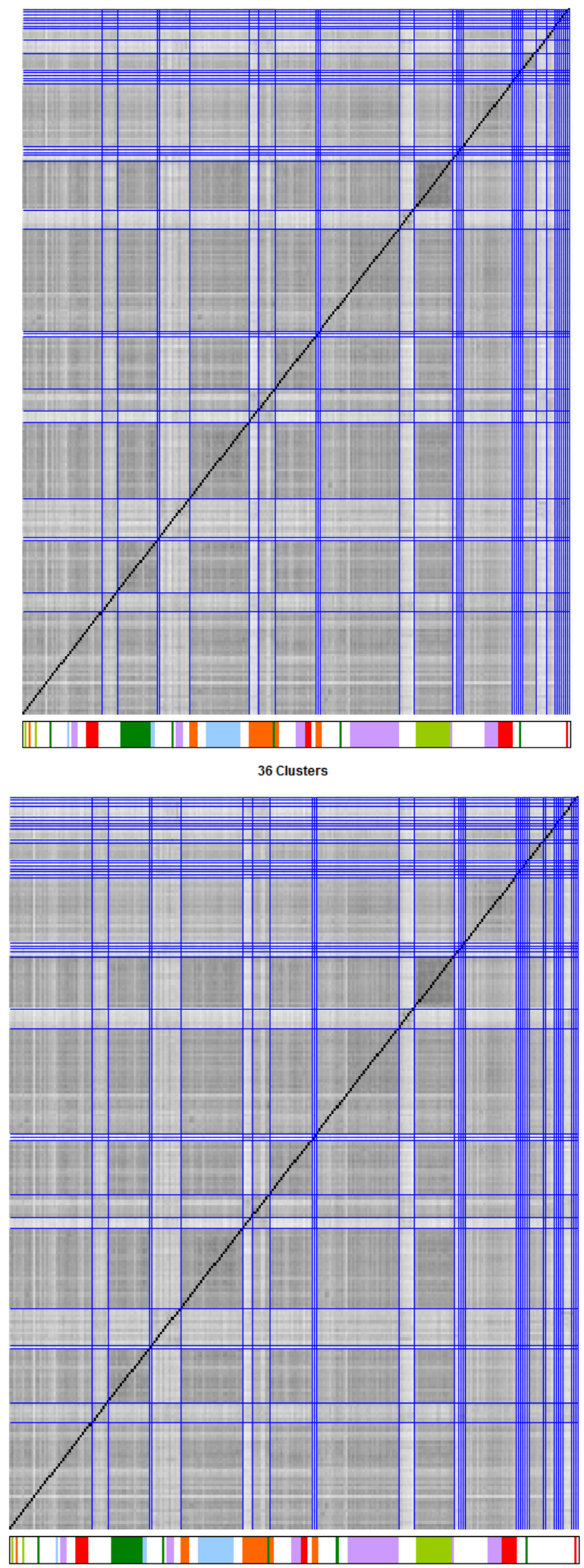

35 Clusters

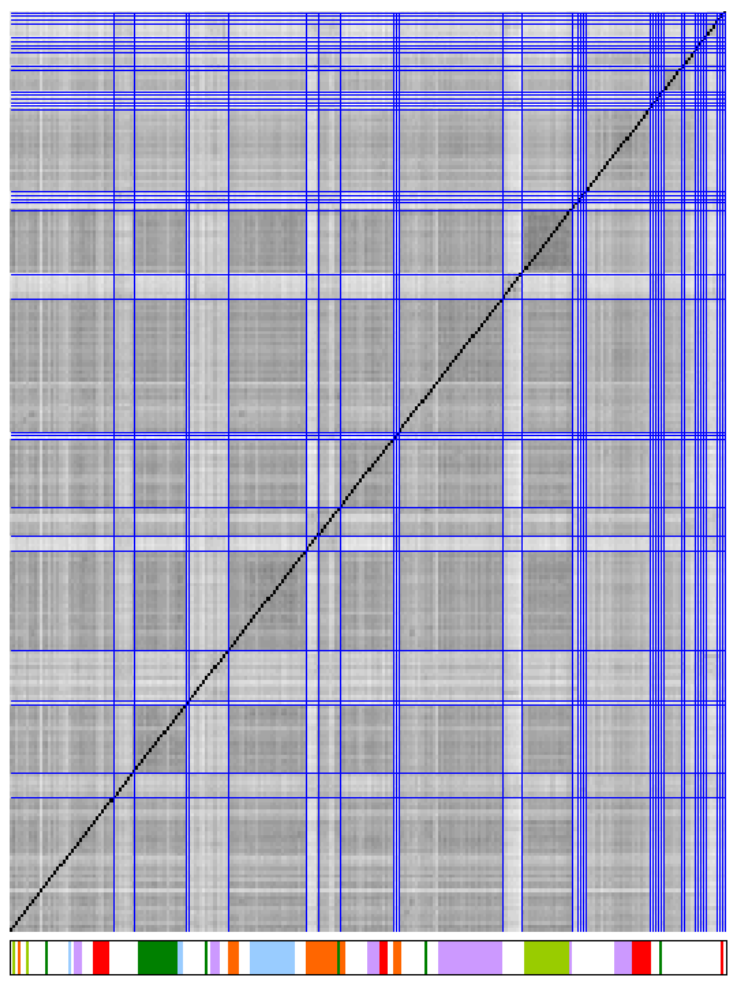

37 Clusters

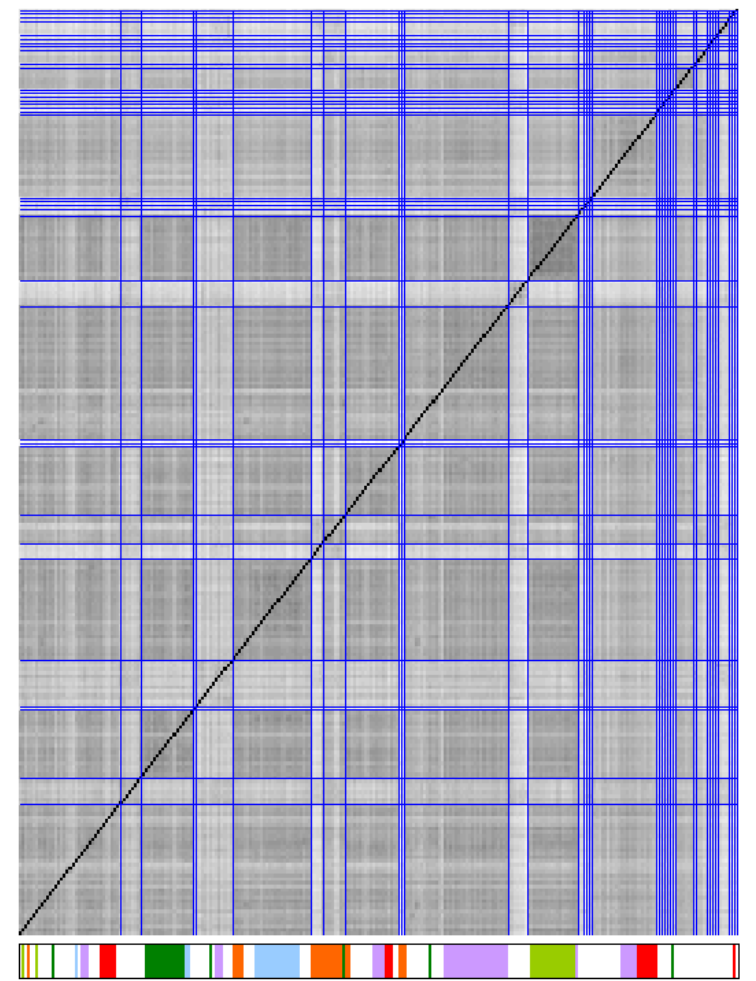


38 Clusters

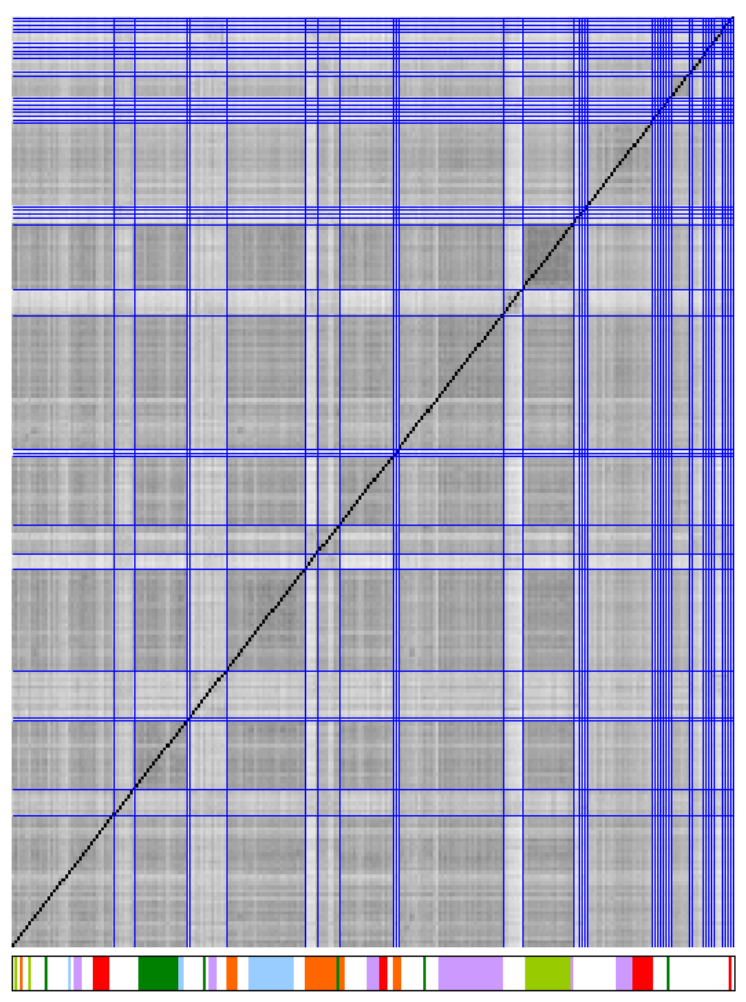

40 Clusters

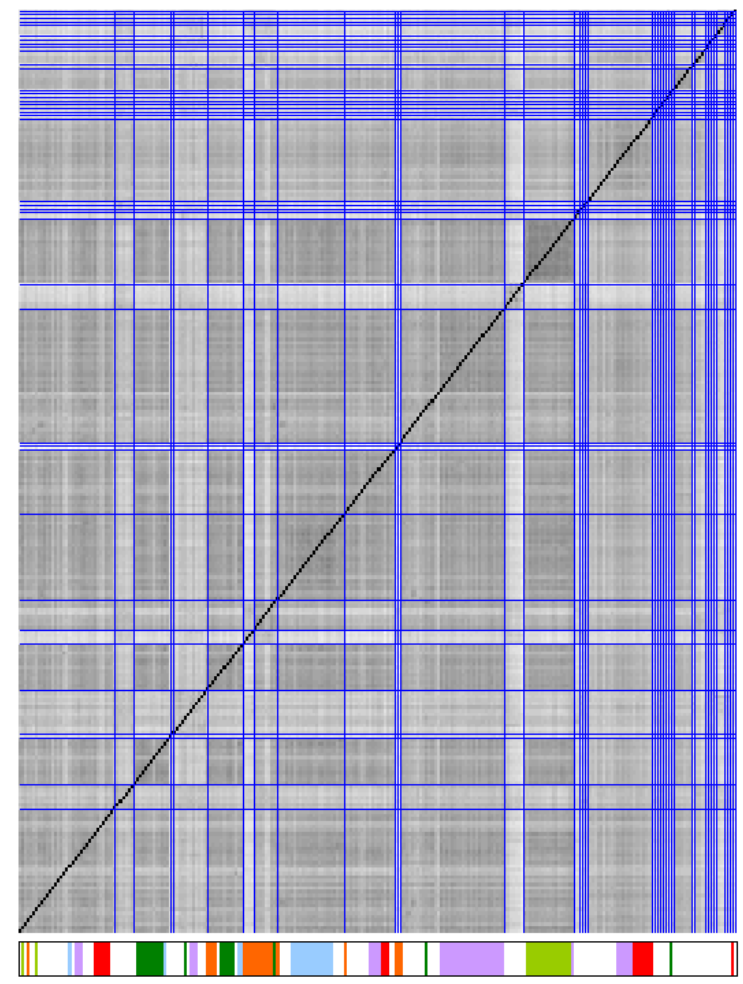

39 Cluster

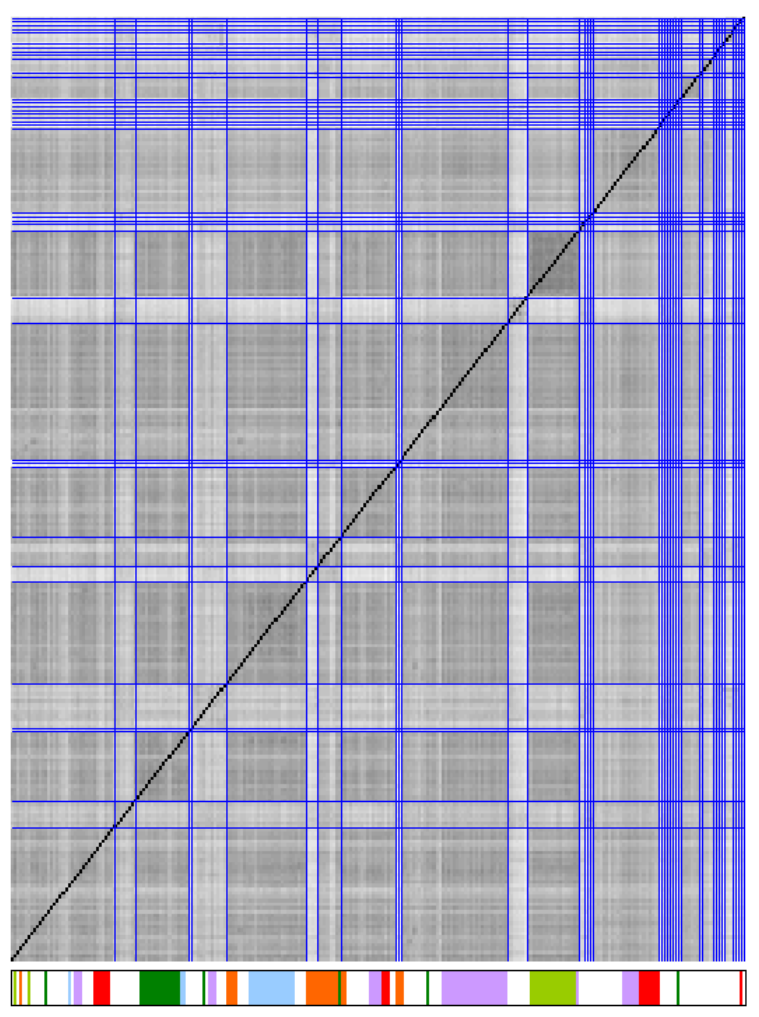

41 Clusters

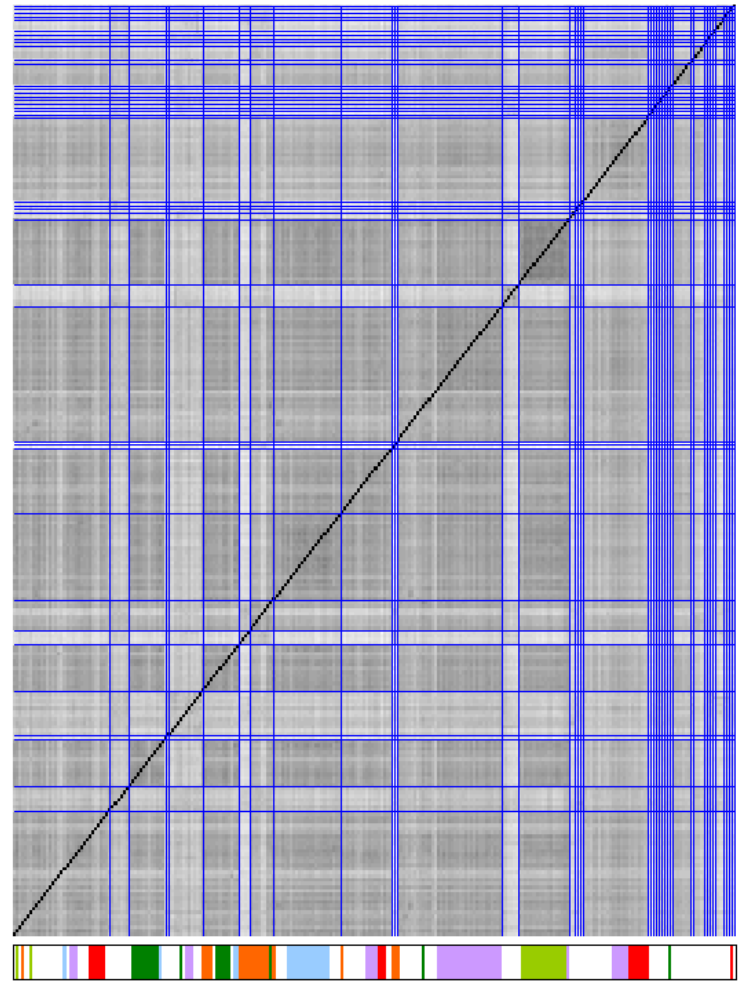


42 Clusters
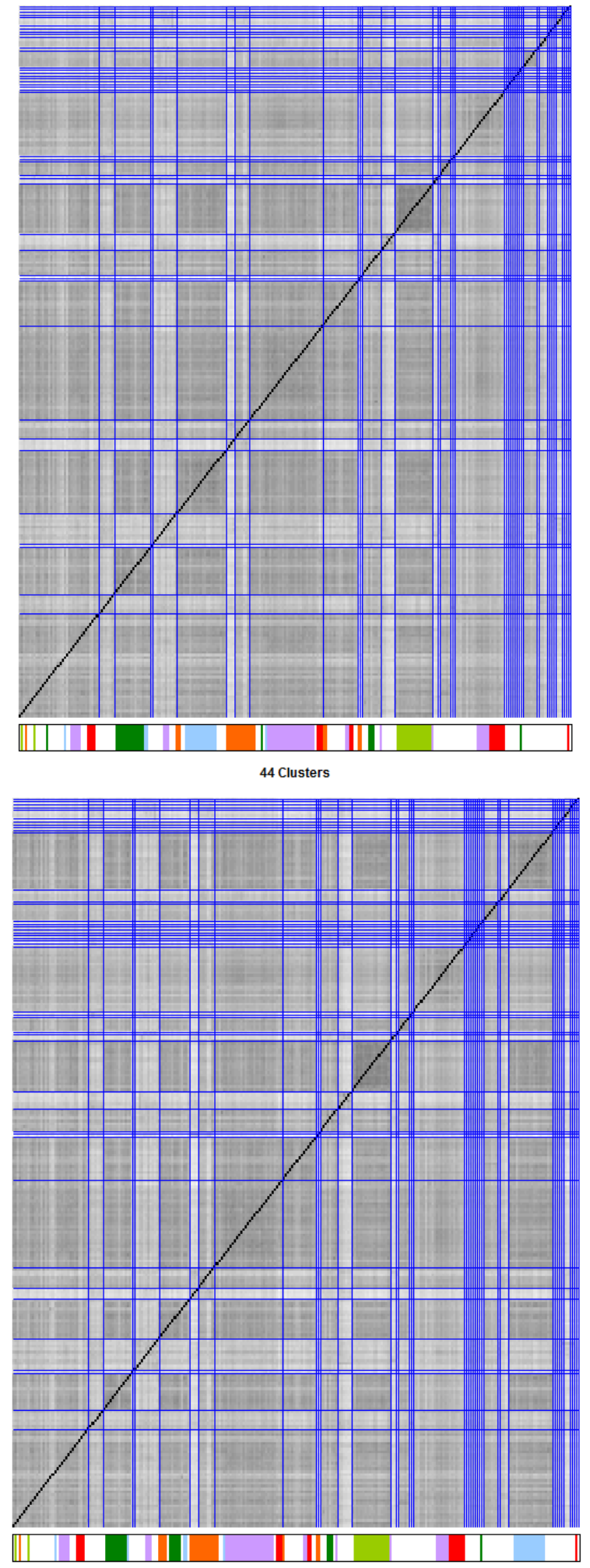

43 Clusters

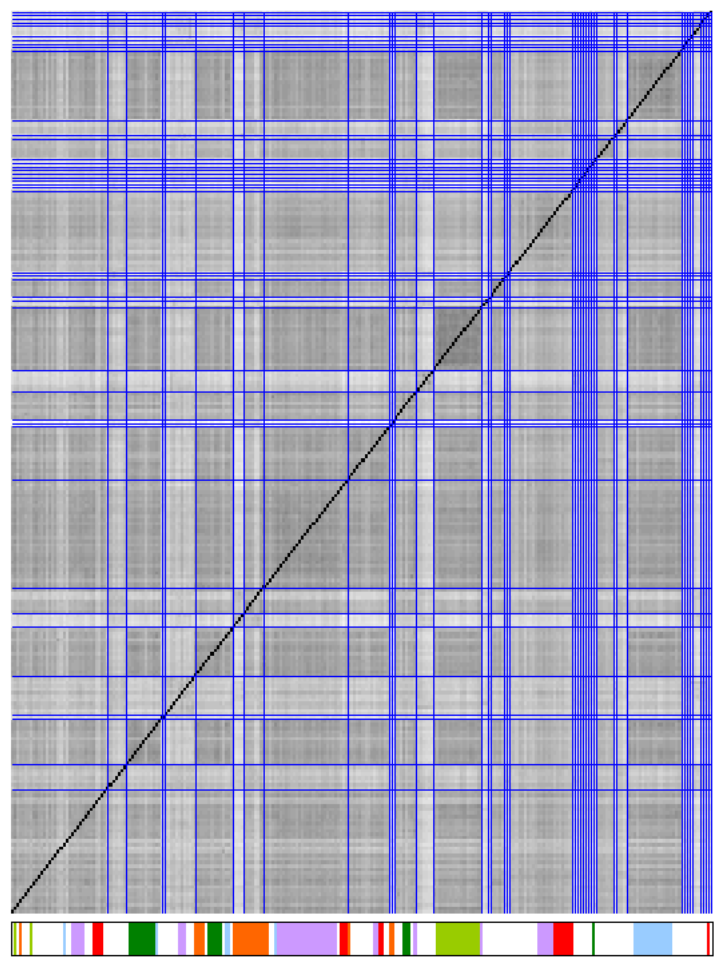

45 Clusters

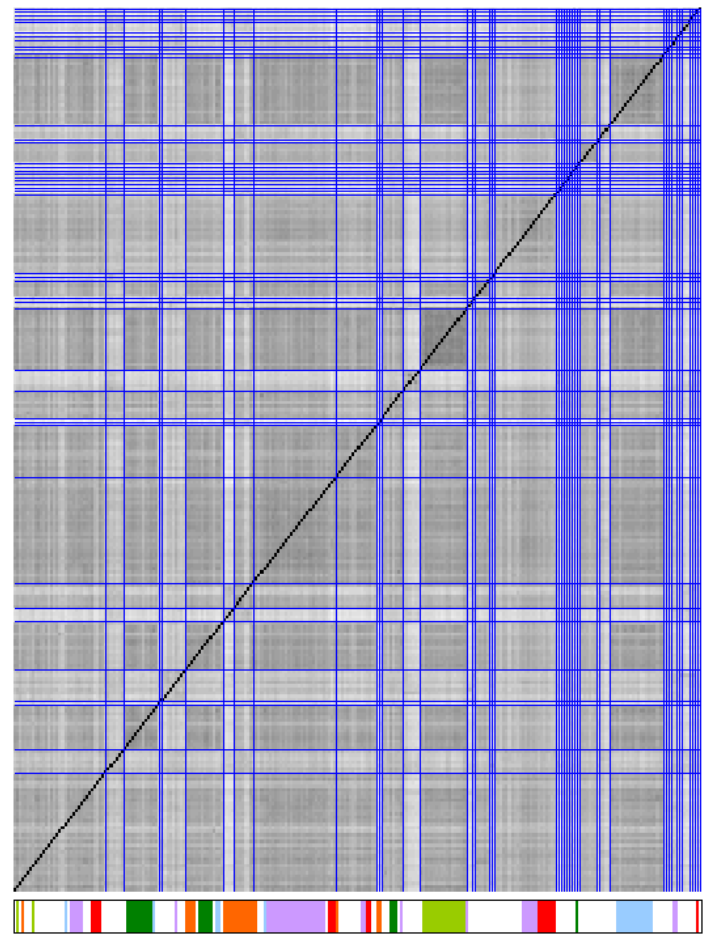


46 Clusters

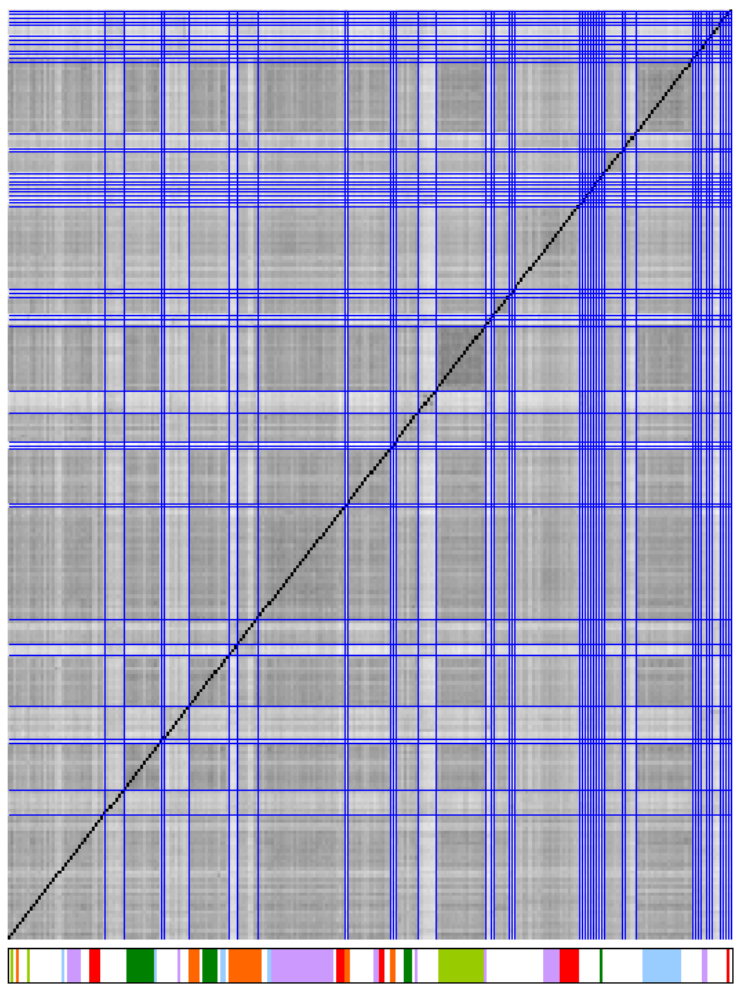

48 Clusters

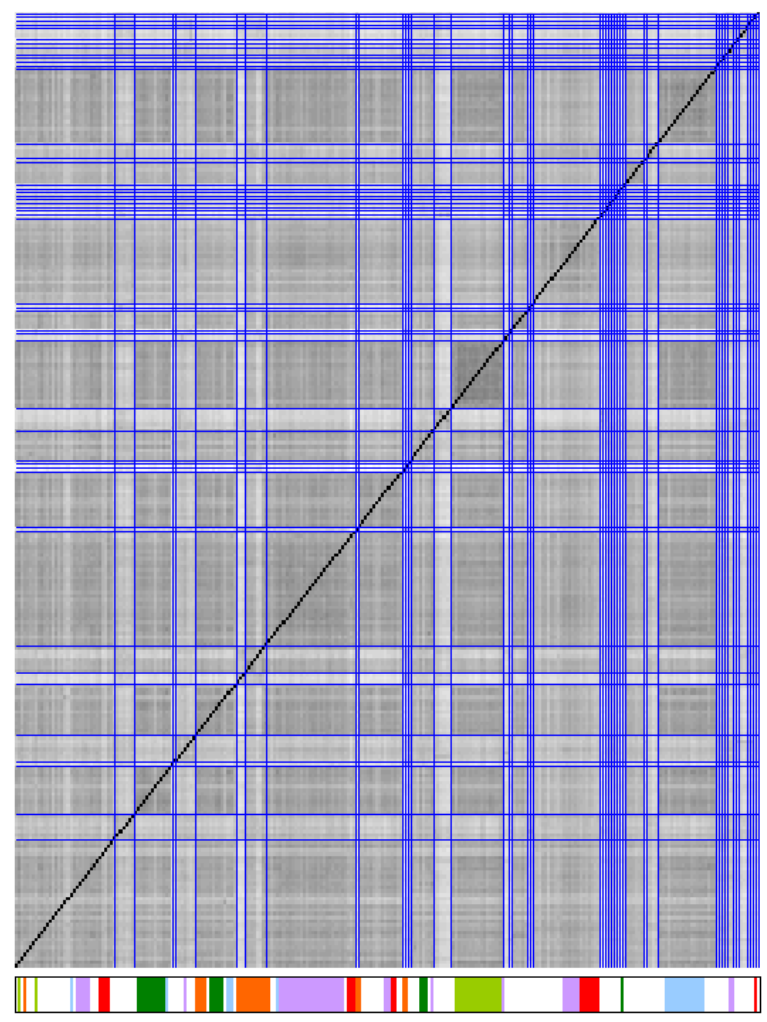

47 Clusters

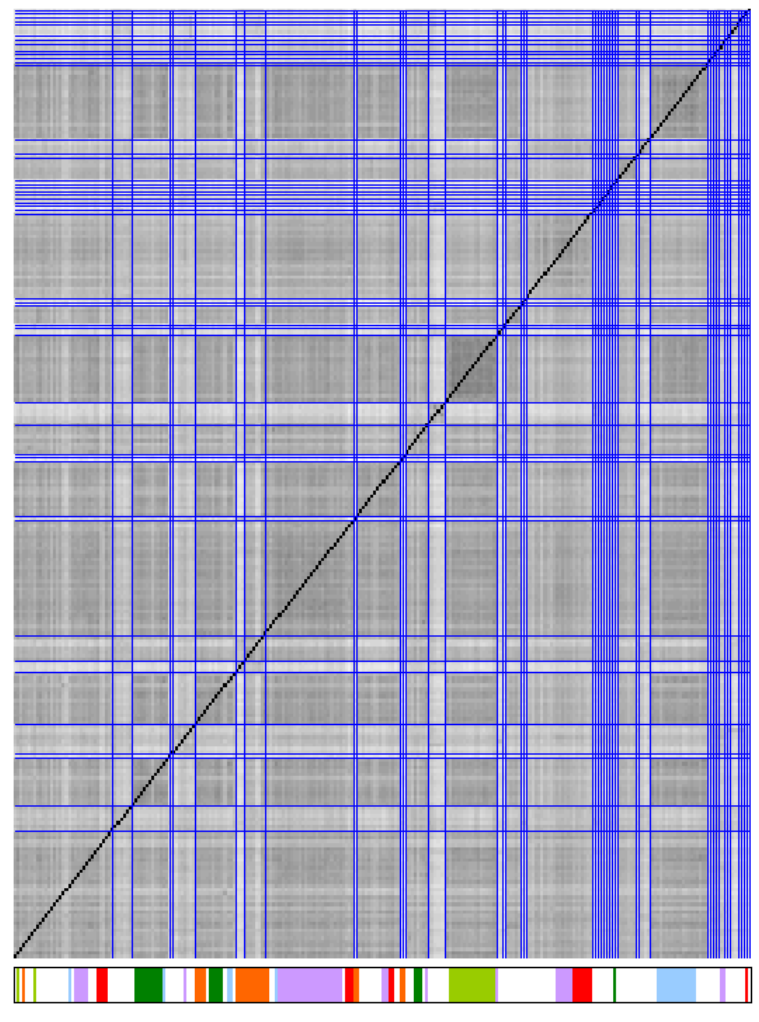

49 Clusters

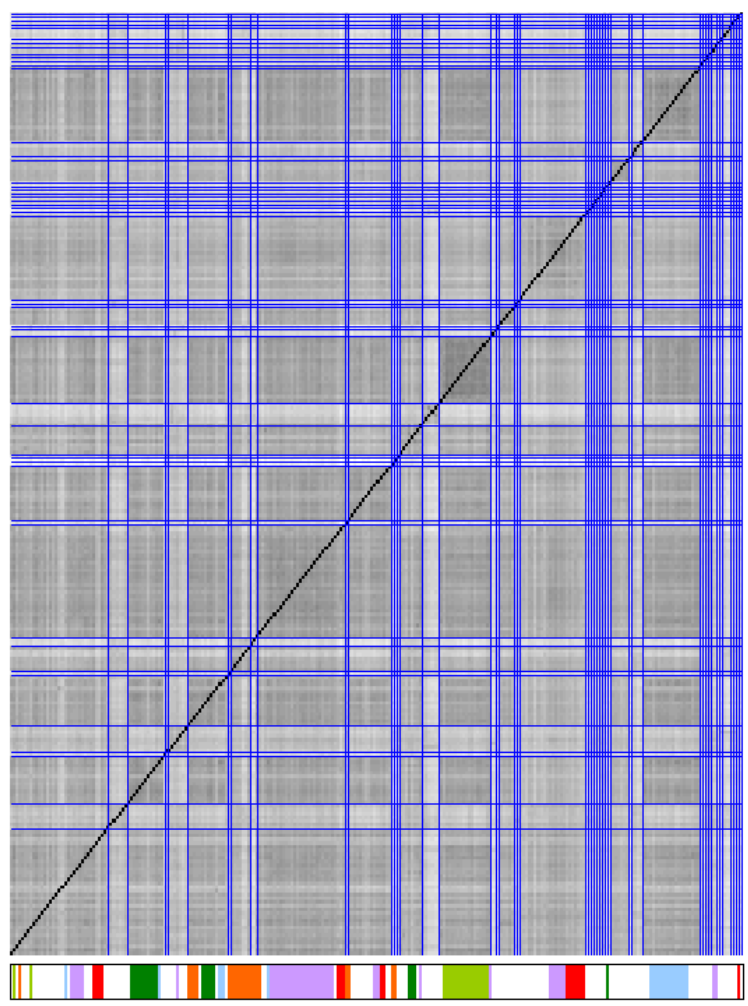




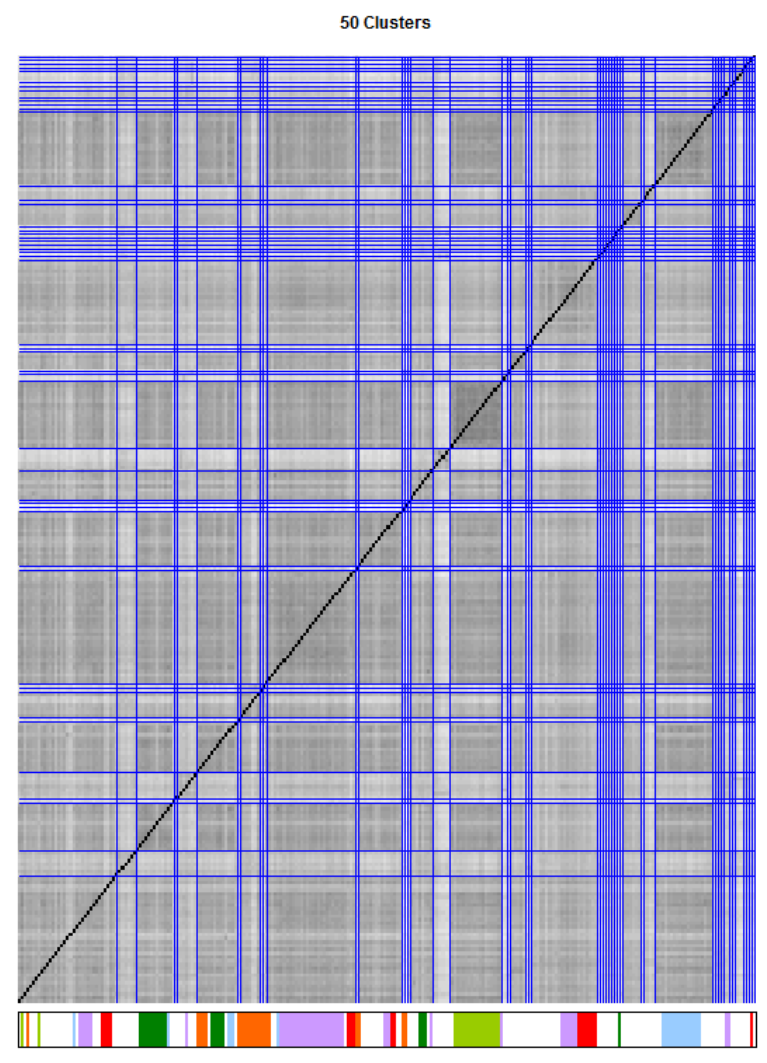

52 Clusters

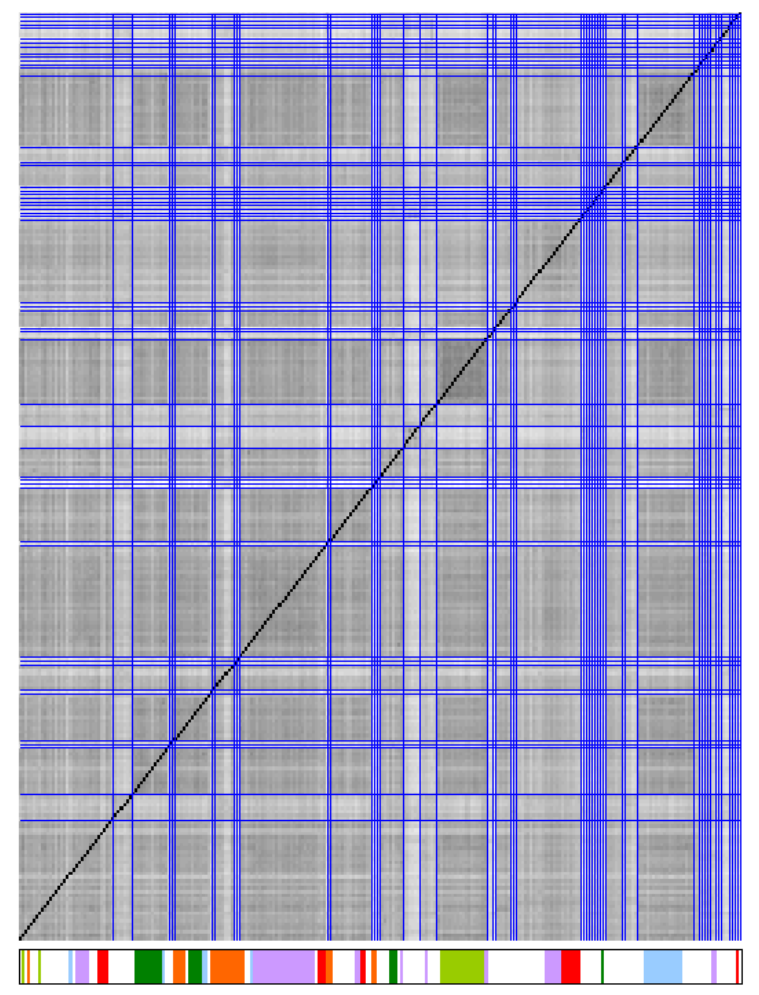

51 Clusters

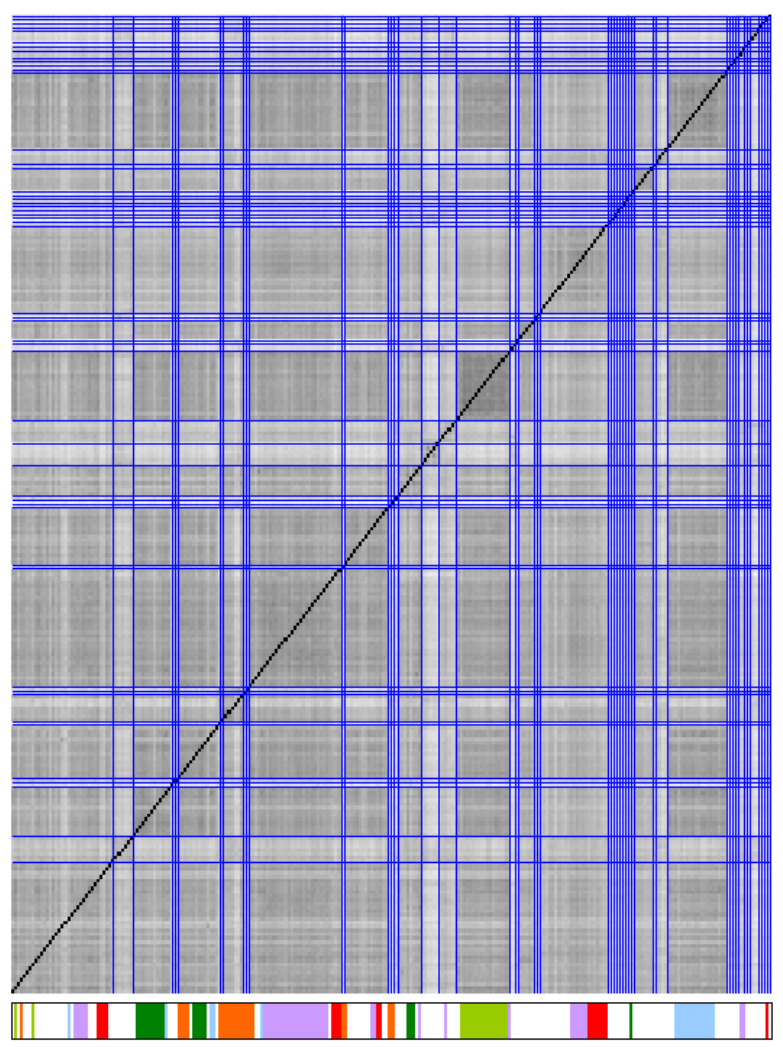

53 Clusters

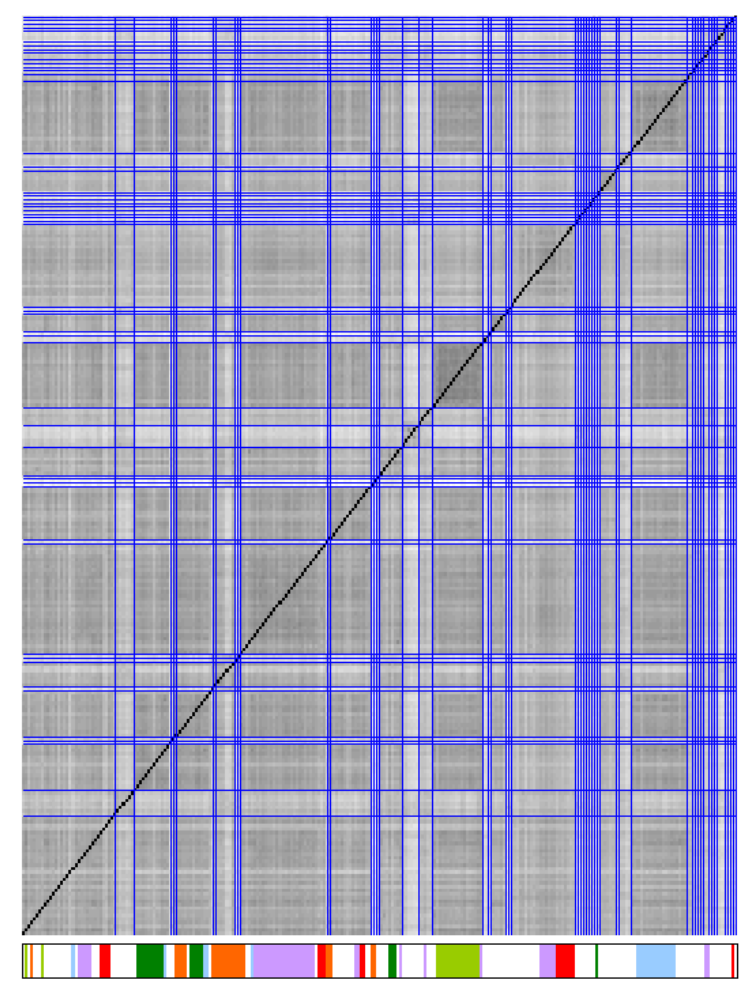


54 Clusters
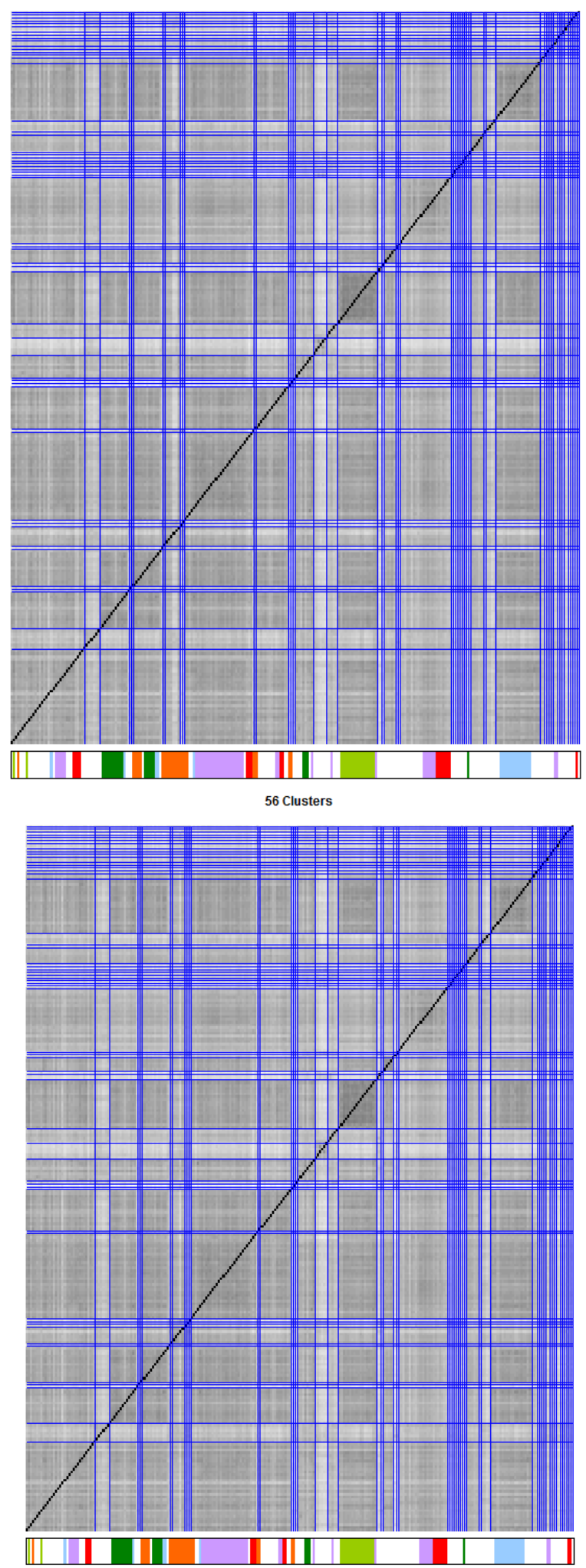

55 Clusters

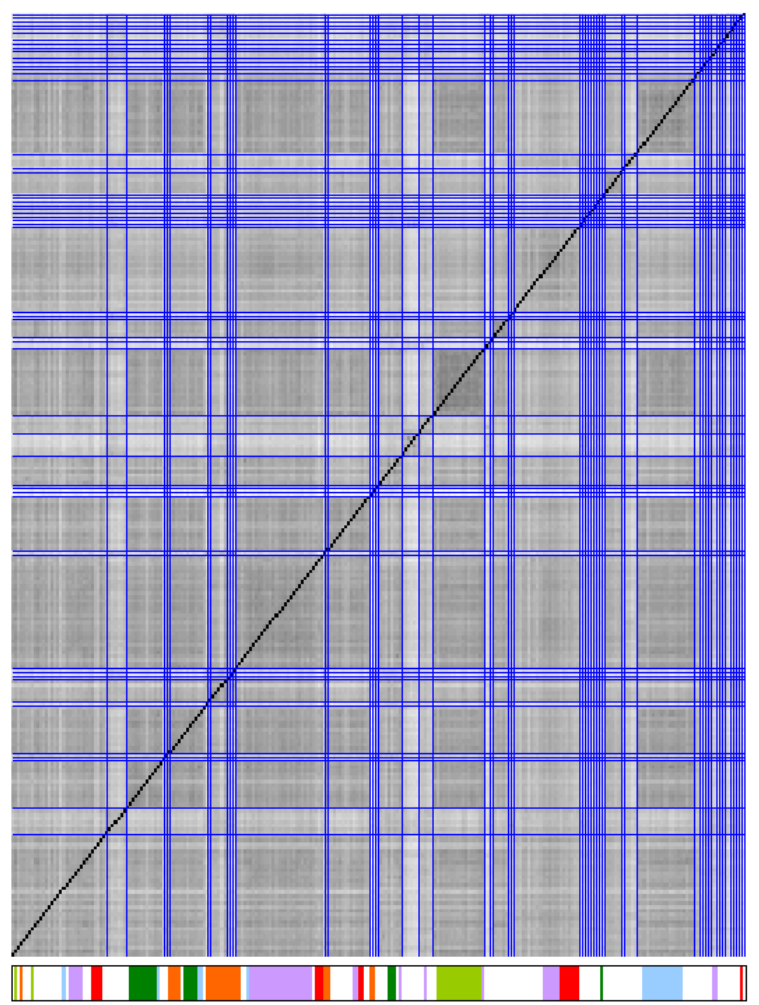

57 Clusters

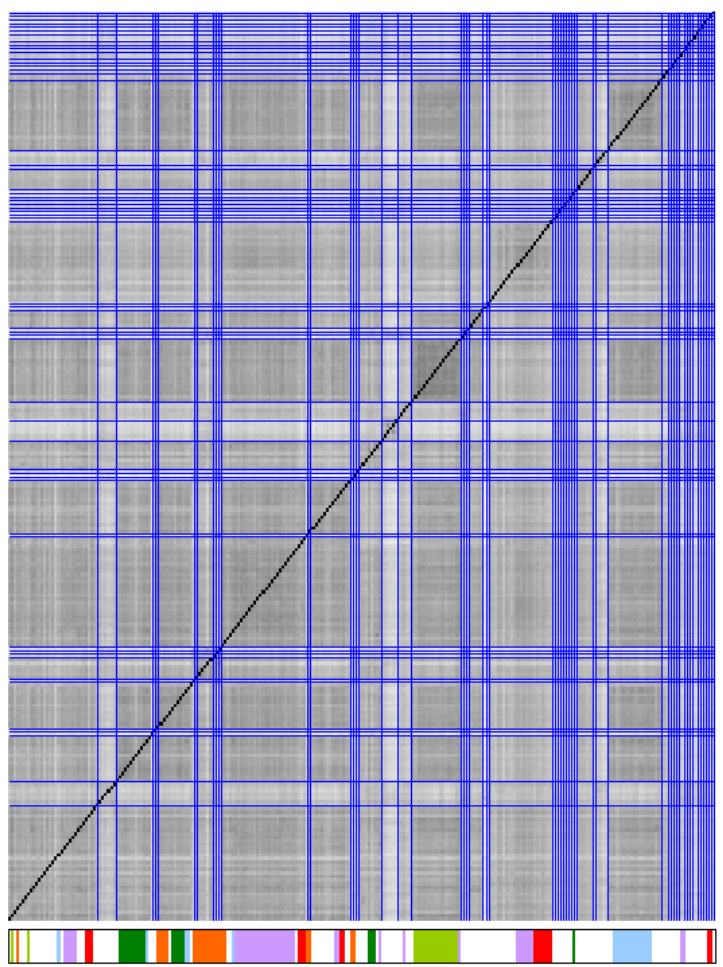



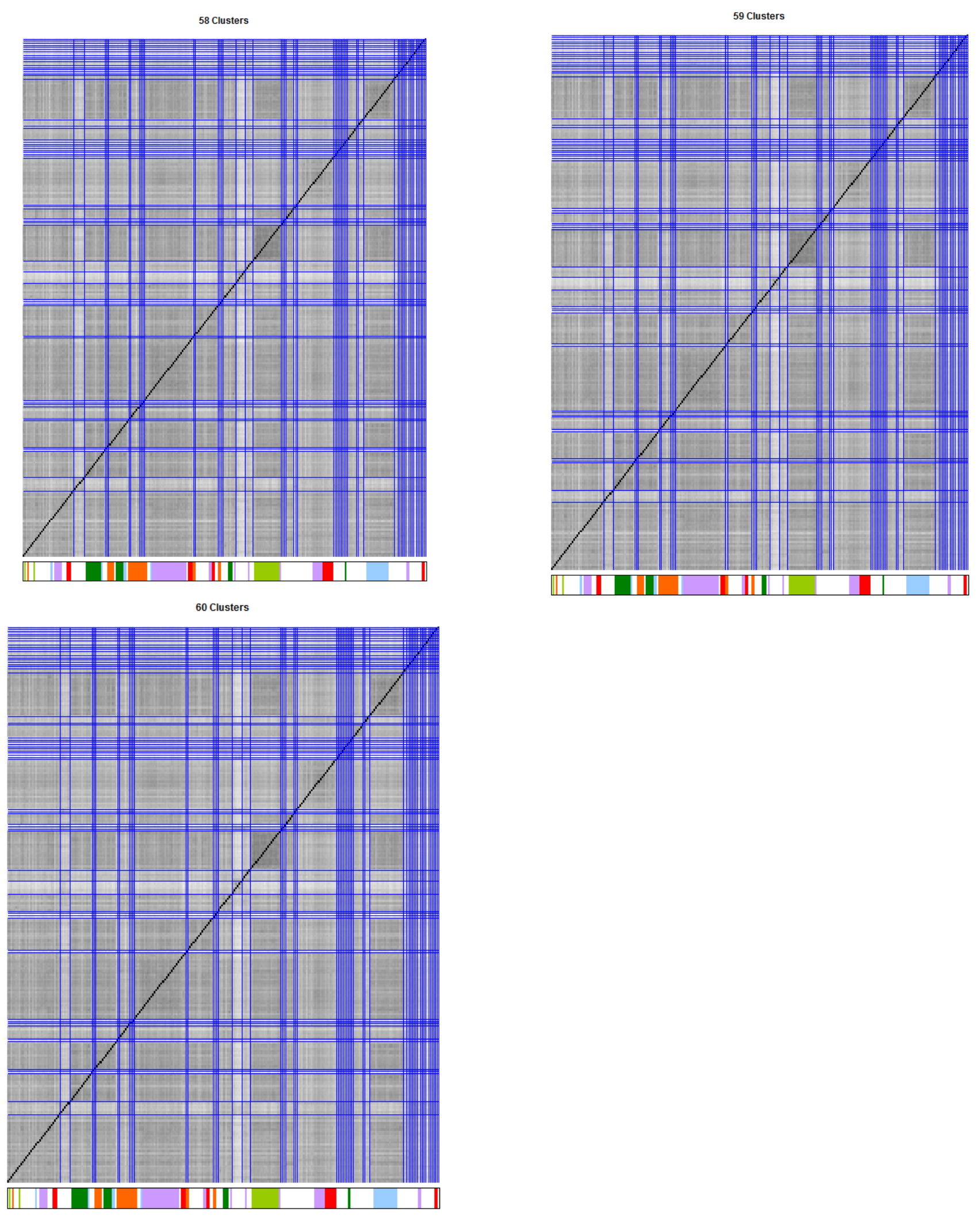